\title{
NOTES ON BEILINSON'S "HOW TO GLUE PERVERSE SHEAVES"
}

\author{
RYAN REICH
}

\begin{abstract}
The titular, foundational work of Beilinson not only gives a technique for gluing perverse sheaves but also implicitly contains constructions of the nearby and vanishing cycles functors of perverse sheaves. These constructions are completely elementary and show that these functors preserve perversity and respect Verdier duality on perverse sheaves. The work also defines a new, "maximal extension" functor, which is left mysterious aside from its role in the gluing theorem. In these notes, we present the complete details of all of these constructions and theorems.
\end{abstract}

In this paper we discuss Alexander Beilinson's "How to glue perverse sheaves" 1 with three goals. The first arose from a suggestion of Dennis Gaitsgory that the author study the construction of the unipotent nearby cycles functor $R \psi^{\text {un }}$ which, as Beilinson observes in his concluding remarks, is implicit in the proof of his Key Lemma 2.1. Here, we make this construction explicit, since it is invaluable in many contexts not necessarily involving gluing. The second goal is to restructure the presentation around this new perspective; in particular, we have chosen to eliminate the two-sided limit formalism in favor of the straightforward setup indicated briefly in $3, \S 4.2]$ for D-modules. We also emphasize this construction as a simple demonstration that $R \psi^{\text {un }}[-1]$ and Verdier duality $\mathbb{D}$ commute, and de-emphasize its role in the gluing theorem. Finally, we provide complete proofs; with the exception of the Key Lemma, 1] provides a complete program of proof which is not carried out in detail, making a technical understanding of its contents more difficult given the density of ideas. This paper originated as a learning exercise for the author, so we hope that in its final form it will be helpful as a learning aid for others. We do not intend it to supplant, but merely to supplement, the original, and we are grateful to Beilinson for his generosity in permitting this.

The author would like to offer three additional thanks: to Gaitsgory, who explained how this beautiful construction can be understood concretely, thus providing the basis for the perspective taken here; to Sophie Morel, for confirming the author's understanding of nearby and vanishing cycles as presented below; and to Mark de Cataldo, for his generous contribution of time and effort to the improvement of these notes.

In order to maintain readability, we will work with sheaves of vector spaces in the classical topology on complex algebraic varieties, except in the second part of Section 4, where we will require the field of coefficients to be algebraically closed. For the necessary modifications to étale sheaves, one should consult Beilinson's paper: aside from the shift in definitions the only change is some Tate twists. For the D-modules case, one should read Sam Lichtenstein's undergraduate thesis, 11, in which the two-sided limit construction is also given in detail. 


\section{TheOrEtiCAL PRELIMINARIES}

The topic at hand is perverse sheaves and nearby cycles; for greater accessibility of these notes, we give a summary of the definitions and necessary properties here.

Diagram chases. Occasionally, we indicate diagram chases in a proof. For ease of reading we have tried not to make this an essential point, but in case the reader should find such a chase to be a convincing informal argument, we indicate here why it is also a convincing formal one.

Every object in an abelian category A can be considered, via Yoneda's lemma, to be a sheaf, namely its functor of points, on the canonical topology of $\mathbf{A}$. This is, by definition, the largest Grothendieck topology in which all representable functors $\operatorname{Hom}_{\mathbf{A}}(\bullet, x)$ are sheaves, and its open covers are precisely the universal strict epimorphisms. Such a map is, in a more general category, a map $f: u \rightarrow x$ such that the fibered product $u^{\prime}=u \times_{x} u$ exists, the coequalizer $x^{\prime}=\operatorname{coker}\left(u^{\prime} \rightrightarrows u\right)$ exists, the natural map $x^{\prime} \rightarrow x$ is an isomorphism, and that all of this is also true when we make any base change along a map $g: y \rightarrow x$, for the induced map $f \times_{x}$ id: $u \times_{x} y \rightarrow y$. In an abelian category, however, this is all equivalent merely to the statement that $f$ is a surjection.

Recall the definitions of the various constructions on sheaves:

(1) Kernels of maps are taken sectionwise; i.e. for a map $f: \mathcal{F} \rightarrow \mathcal{G}, \operatorname{ker}(f)(U)=$ $\operatorname{ker}(f(U): \mathcal{F}(U) \rightarrow \mathcal{G}(U))$. Likewise, products and limits are taken sectionwise.

(2) Cokernels are locally taken sectionwise: any section $s \in \operatorname{coker}(f)(U)$ is, on some open cover $V$ of $U$, of the form $\bar{t}$ for $t \in \mathcal{G}(V)$. Likewise, images, coproducts, and colimits are taken locally.

In an abelian category, where all of these constructions exist by assumption, these descriptions are even prescriptive: if one forms the sheaves thus described, they are representable by the objects claimed. Therefore, the following common arguments in diagram chasing are valid:

(1) A map $f: x \rightarrow y$ is surjective if and only if for every $s \in y$, there is some $t \in x$ such that $s=f(t)$. This is code for: for every "open set" $U$ and every $s \in y(U)$, there is a surjection $V \rightarrow U$ and a section $t \in y(V)$ such that $\left.s\right|_{V}=f(t)$.

(2) If $s \in y$, then $\bar{s}=0 \in \operatorname{coker}(f)$ if and only if $s \in \operatorname{im}(f)$. This is code for: if $s \in y(U)$ and $\bar{s}=0 \in \operatorname{coker}(f)(U)$, then there is some surjection $V \rightarrow U$ and $t \in x(V)$ with $\left.s\right|_{V}=f(t)$.

(3) For $s, t \in x, s=t$ if and only if $s-t=0$. Here, the sum of maps $s: U \rightarrow x$ and $t: V \rightarrow x$ is obtained by forming the fibered product $W=$ $U \times{ }_{x} V$ which covers both $U$ and $V$, and then taking the sum of the maps $\left.s\right|_{W},\left.t\right|_{W} \in \operatorname{Hom}(W, x)$; the condition for equality is just the statement that a section of a sheaf vanishes if only it vanishes on an open cover.

Any other arguments involving elements and some concept related to exactness can also be phrased in this way. Thus, a naïve diagram-chasing argument can be converted into a rigorous one simply by replacing statements like $s \in x$ with correct ones $s \in x(U)$ for some open set $U$, and passing to surjective covers of $U$ when necessary. 
Derived category and functors. All the action takes place in the derived category; specifically, let $X$ be an algebraic variety and denote by $\mathbf{D}(X)$ its derived category of bounded complexes of sheaves of vector spaces with constructible cohomology. By definition, a map of complexes $f: A^{\bullet} \rightarrow B^{\bullet}$ defines an isomorphism in $\mathbf{D}(X)$ if and only if its associated map on cohomology sheaves $H^{i}(f): H^{i}\left(A^{\bullet}\right) \rightarrow$ $H^{i}\left(B^{\bullet}\right)$ is an isomorphism for all $i$. We have a notation for the index-shift: $A^{i+1}=(A[1])^{i}$ (technically, the differential maps also change sign, but we will never need to think about this). The derived category $\mathbf{D}(X)$ is a "triangulated category", which means merely that in it are a class of triples, called "distinguished triangles", of complexes and maps

$$
A^{\bullet} \rightarrow B^{\bullet} \rightarrow C^{\bullet} \rightarrow A^{\bullet}[1]
$$

in which two consecutive arrows compose to zero, satisfying the axioms given in, for example, 9] (but see also Section 4), and with the property that the associated long sequence of cohomology sheaves

$$
\ldots H^{-1}\left(C^{\bullet}\right) \rightarrow H^{0}\left(A^{\bullet}\right) \rightarrow H^{0}\left(B^{\bullet}\right) \rightarrow H^{0}\left(C^{\bullet}\right) \rightarrow H^{1}\left(A^{\bullet}\right) \rightarrow \ldots
$$

is exact (note that $H^{0}\left(A^{\bullet}[1]\right)=H^{1}\left(A^{\bullet}\right)$ ); we say that the $H^{i}$ are "cohomological". If $f: A^{\bullet} \rightarrow B^{\bullet}$ is given, there always exists a triangle whose third term $C^{\bullet}=$ Cone $(f)$ is the "cone" of $f$; this cone is unique up to nonunique isomorphism and any commutative diagram of maps $f$ induces a map on cones, but this is not functorial. It follows that the induced triangle itself is unique up to a nonunique isomorphism whose component morphisms on $A^{\bullet}$ and $B^{\bullet}$ are the identity maps. A functor between two triangulated categories is "triangulated" if it sends triangles in one to triangles in the other.

In $\mathbf{D}(X)$ we also have some standard constructions of sheaf theory. For any two complexes there is the "total tensor product" $A^{\bullet} \otimes B^{\bullet}$ obtained by taking in degree $n$ the direct sum of all products $A^{i} \otimes B^{j}$ with $i+j=n$ (and some differentials that are irrelevant) and its derived bifunctor $A^{\bullet} \otimes^{L} B^{\bullet}$, with $H^{i}\left(A^{\bullet} \otimes^{L} B^{\bullet}\right)=$ $\operatorname{Tor}^{i}\left(A^{\bullet}, B^{\bullet}\right)$, which is a triangulated functor in each variable. We also have the bifunctor (contravariant in the first argument) $\mathcal{H o m}\left(A^{\bullet}, B^{\bullet}\right)$, whose terms are $\mathcal{H} \mathrm{om}\left(A^{\bullet}, B^{\bullet}\right)^{i}(U)=\operatorname{Hom}\left(\left.A^{\bullet}\right|_{U},\left.B^{\bullet}[i]\right|_{U}\right)$, and its derived bifunctor $R \mathcal{H} \mathrm{om}\left(A^{\bullet}, B^{\bullet}\right)$, with $H^{i} R \mathcal{H o m}\left(A^{\bullet}, B^{\bullet}\right)=\operatorname{Ext}^{i}\left(A^{\bullet}, B^{\bullet}\right)$, which is triangulated in each variable. Of course, these two have an adjunction:

$$
R \mathcal{H o m}\left(A^{\bullet} \stackrel{L}{\otimes} B^{\bullet}, C^{\bullet}\right) \cong R \mathcal{H} \operatorname{om}\left(A^{\bullet}, R \mathcal{H} \operatorname{om}\left(B^{\bullet}, C^{\bullet}\right)\right) .
$$

For any Zariski-open subset $U \subset X$ with inclusion map $j$, there are triangulated functors $j_{!}, j_{*}: \mathbf{D}(U) \rightarrow \mathbf{D}(X)$ and $j^{*}=j^{!}: \mathbf{D}(X) \rightarrow \mathbf{D}(U)$; if $i$ is the inclusion of its complement $Z$, then there are likewise maps $i^{!}, i^{*}: \mathbf{D}(X) \rightarrow \mathbf{D}(Z)$ and $i_{*}=$ $i_{!}: \mathbf{D}(Z) \rightarrow \mathbf{D}(X)$. (Technically the operation $j_{*}$ is only left exact on sheaves and we should write $R j_{*}$ for its derived functor, but we will never have occasion to use the plain version so we elide this extra notation.) They satisfy a number of important relations, of which we will only use one here: there is a functorial triangle in the complex $A_{X}^{\bullet} \in \mathbf{D}(X)$ :

$$
j_{!} j^{*}\left(A_{X}^{\bullet}\right) \rightarrow A_{X}^{\bullet} \rightarrow i_{*} i^{*}\left(A_{X}^{\bullet}\right) \rightarrow
$$

We will generally forget about writing $i_{*}$ and consider $\mathbf{D}(Z) \subset \mathbf{D}(X)$. 
There is also a triangulated duality functor $\mathbb{D}: \mathbf{D}(X) \rightarrow \mathbf{D}(X)^{\text {op }}$ which interchanges ! and $*$, in that $\mathbb{D} j_{*}\left(A_{U}^{\bullet}\right)=j_{!}\left(\mathbb{D} A_{U}^{\bullet}\right)$, etc., and is an involution. In fact, if we set $\mathcal{D}_{X}^{\bullet}=\mathbb{D} \underline{\mathbb{C}}$, then $\mathbb{D}\left(A^{\bullet}\right)=R \mathcal{H o m}\left(A^{\bullet}, \mathcal{D}_{X}^{\bullet}\right)$.

For any map $f: X \rightarrow Y$ of varieties, we have $f^{!}, f^{*}$ as well (also $f_{!}, f_{*}$, and none of them are equal), with the same relationships to $\mathbb{D}$, and the useful identity

$$
f^{!} R \mathcal{H o m}\left(A_{Y}^{\bullet}, B_{Y}^{\bullet}\right)=R \mathcal{H o m}\left(f^{*} A_{Y}^{\bullet}, f^{!} B_{Y}^{\bullet}\right) .
$$

Note that by these properties, we have $f^{!} \mathcal{D}_{Y}^{\bullet}=\mathcal{D}_{X}^{\bullet}$.

Perverse sheaves. Here we give a detail-free overview of the formalism of perverse sheaves created in 4]. Within $\mathbf{D}(X)$ there is an abelian category $\mathbf{M}(X)$ of "perverse sheaves" which has nicer properties than the category of actual sheaves. It is specified by means of a "t-structure", namely, a pair of full subcategories ${ }^{p} \mathbf{D}(X) \leqslant 0$ and ${ }^{p} \mathbf{D}(X) \geqslant 0$, also satisfying some conditions we won't use, and such that

$$
\mathbf{M}(X)={ }^{p} \mathbf{D}(X)^{\leqslant 0} \cap{ }^{p} \mathbf{D}(X)^{\geqslant 0} .
$$

There are truncation functors $\tau^{\leqslant 0}: \mathbf{D}(X) \rightarrow{ }^{p} \mathbf{D}(X) \leqslant 0$ and likewise for $\tau^{\geqslant 0}$, fitting into a distinguished triangle for any complex $A_{X}^{\bullet} \in \mathbf{D}(X)$ :

$$
\tau^{\leqslant 0} A_{X}^{\bullet} \rightarrow A_{X}^{\bullet} \rightarrow \tau^{>0} A_{X}^{\bullet} \rightarrow
$$

(where $\tau^{>0}=\tau^{\geqslant 1}=[-1] \circ \tau^{\geqslant 0} \circ[1]$ ). This triangle is unique with respect to the property that the first term is in ${ }^{p} \mathbf{D}(X)^{\leqslant 0}$ and the third is in ${ }^{p} \mathbf{D}(X)^{>0}$. They have the obvious properties implied by the notation: $\tau^{\leqslant a} \tau^{\leqslant b}=\tau \leqslant a$ if $a \leq b$, and likewise for $\tau^{\geqslant}$?. Furthermore, there are "perverse cohomology" functors ${ }^{p} H^{i}: \mathbf{D}(X) \rightarrow$ $\mathbf{M}(X)$, where of course ${ }^{p} H^{i}\left(A^{\bullet}\right)={ }^{p} H^{0}\left(A^{\bullet}[i]\right)$ and ${ }^{p} H^{0}=\tau^{\geqslant 0} \tau^{\leqslant 0}=\tau^{\leqslant 0} \tau^{\geqslant 0}$; these are cohomological just like the ordinary cohomology functors. The abelian category structure of $\mathbf{M}(X)$ is more or less determined by the fact that if we have a map $f: \mathcal{F} \rightarrow \mathcal{G}$ of perverse sheaves (this is the notation we will be using; we will not think of perverse sheaves as complexes), then

$$
\text { ker } f={ }^{p} H^{-1} \text { Cone }(f) \quad \text { coker } f={ }^{p} H^{0} \text { Cone }(f) .
$$

For notational convenience, we will write $\mathcal{M}$ for a perverse sheaf on $U, \mathcal{F}$ for one on $X$, and as usual, abandon $i_{*}$ and just consider $\mathbf{M}(Z) \subset \mathbf{M}(X)$ (for the reason expressed immediately below, this is reasonable).

The category $\mathbf{M}(X)$ is closed under the duality functor $\mathbb{D}$, but not necessarily under the six functors defined for an open/closed pair of subvarieties. However, it is true that $j_{*}(\mathcal{M}), i^{!}(\mathcal{F}) \in{ }^{p} \mathbf{D}^{\geqslant 0}$ and $j_{!}(\mathcal{M}), i^{*}(\mathcal{F}) \in{ }^{p} \mathbf{D}^{\leqslant 0}$, while $j^{*}(\mathcal{F}), i_{*}\left(\mathcal{F}_{Z}\right) \in \mathbf{M}$ $\left(\mathcal{F}_{Z}\right.$ a perverse sheaf on $\left.Z\right)$; we say these functors are right, left, or just "t-exact". Furthermore, when $j$ is an affine morphism (the primary example being when $Z$ is a Cartier divisor), both $j_{\text {! }}$ and $j_{*}$ are t-exact, and thus their restriction to $\mathbf{M}(U)$ is exact with values in $\mathbf{M}(X)$. There is also a "minimal extension" functor $j_{!_{*}}$, defined so that $j_{!_{*}}(\mathcal{M})$ is the image of ${ }^{p} H^{0}\left(j_{!} \mathcal{M}\right)$ in ${ }^{p} H^{0}\left(j_{*} \mathcal{M}\right)$ along the natural map $j_{!} \rightarrow j_{*}$; it is the unique perverse sheaf such that $i^{*} j_{!_{*}} \mathcal{M} \in{ }^{p} \mathbf{D}^{<0}(Z)$ and $i^{!} j_{! *} \mathcal{M} \in{ }^{p} \mathbf{D}^{>0}(Z)$, but for us the most useful property is that when $j$ is an affine, open immersion, then we have a sequence of perverse sheaves

$$
i^{*} j_{!_{*}} \mathcal{M}[-1] \hookrightarrow j_{!} \mathcal{M} \rightarrow j_{!_{*}} \mathcal{M} \hookrightarrow j_{*} \mathcal{M} \rightarrow i^{!} j_{!_{*}} \mathcal{M}[1]
$$

i.e. $i^{*} j_{!_{*}} \mathcal{M}[-1]=\operatorname{ker}\left(j_{!} \mathcal{M} \rightarrow j_{*} \mathcal{M}\right)$ and $i^{!} j_{!_{*}} \mathcal{M}[1]=\operatorname{coker}\left(j_{!} \mathcal{M} \rightarrow j_{*} \mathcal{M}\right)$ are both perverse sheaves. 
Perverse sheaves have good category-theoretic properties: $\mathbf{M}(X)$ is both artinian and noetherian, so every perverse sheaf has finite length. Finally, we will use the sheaf-theoretic fact that if $\mathcal{L}$ is a locally constant sheaf on $X$, then $\mathcal{F} \otimes \mathcal{L}$ is perverse whenever $\mathcal{F}$ is. Note that since $\mathcal{L}$ is locally free, it is flat, and therefore $\mathcal{F} \otimes \mathcal{L}=\mathcal{F} \otimes^{L} \mathcal{L}$.

Nearby cycles. If we have a map $f: X \rightarrow \mathbb{A}^{1}$ such that $Z=f^{-1}(0)$ (so $U=$ $\left.f^{-1}\left(\mathbb{A}^{1} \backslash\{0\}\right)=f^{-1}\left(\mathbf{G}_{\mathbf{m}}\right)\right)$, the "nearby cycles" functor $R \psi_{f}: \mathbf{D}(U) \rightarrow \mathbf{D}(Z)$ is defined. Namely, let $u: \widetilde{\mathbf{G}_{\mathbf{m}}} \rightarrow \mathbf{G}_{\mathbf{m}}$ be the universal cover of $\mathbf{G}_{\mathbf{m}}=\mathbb{A}^{1} \backslash\{0\}$, let $v: \widetilde{U}=U \times \mathbf{G}_{\mathbf{m}} \widetilde{\mathbf{G}_{\mathbf{m}}} \rightarrow U$ be its pullback, forming a diagram

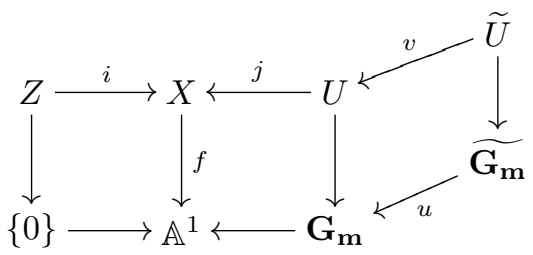

and set (in this one instance, explicitly writing $j_{*}$ and $v_{*}$ as non-derived functors)

$$
R \psi_{f}=R\left(i^{*} j_{*} v_{*} v^{*}\right): \mathbf{D}(U) \rightarrow \mathbf{D}(Z) .
$$

Since $i^{*}$ and $v^{*}$ are exact, indeed $\psi_{f}$ is a left-exact functor from sheaves on $U$ to sheaves on $Z$. Many sources (e.g. [13, $\S 1.1 .1]$ ) give the definition $R \psi_{f}=$ $i^{*} R j_{*} R v_{*} v^{*}$; in fact, they are the same: since $v$ is a covering map, if $\mathcal{F}$ is a flasque sheaf on $U$, then $v^{*} \mathcal{F}$ is flasque on $\widetilde{U}$ and so acyclic for $v_{*}$ (and $v_{*} v^{*} \mathcal{F}$ acyclic for $\left.j_{*}\right)$. Therefore we may form the derived functor before or after composition. Note that $v$ is not an algebraic map, and therefore it is not a priori clear whether $R \psi_{f}$ preserves constructibility; that it does is a theorem of Deligne ([7], Exposé XIII, Théorème 2.3 for étale sheaves and Exposé XIV, Théorème 2.8 for the comparison with classical nearby cycles).

The fundamental group $\pi_{1}\left(\mathbf{G}_{\mathbf{m}}\right)$ acts on any $v^{*} A_{U}^{\bullet}$ via deck transformations of $\widetilde{\mathbf{G}_{\mathbf{m}}}$ and therefore acts on $\psi_{f}$ and $R \psi_{f}$. There is a natural map $i^{*} A_{X}^{\bullet} \rightarrow \psi_{f}\left(j^{*} A_{X}^{\bullet}\right)$, obtained from $\left(v^{*}, v_{*}\right)$-adjunction, on whose image $\pi_{1}\left(\mathbf{G}_{\mathbf{m}}\right)$ acts trivially. We set, by definition,

$$
i^{*} A_{X}^{\bullet} \rightarrow \psi_{f}\left(j^{*} A_{X}^{\bullet}\right) \rightarrow \phi_{f}\left(A_{X}^{\bullet}\right) \rightarrow 0
$$

where $\phi_{f}\left(A_{X}^{\bullet}\right)$ is the "vanishing cycles" sheaf. Using some homological algebra tricks the above sequence induces a natural distinguished triangle

$$
i^{*} A_{X}^{\bullet} \rightarrow R \psi_{f}\left(j^{*} A_{X}^{\bullet}\right) \rightarrow R \phi_{f}\left(A_{X}^{\bullet}\right) \rightarrow
$$

where $R \phi_{f}$ is (morally) the right derived functor of $\phi_{f}$. Like $R \psi_{f}, R \phi_{f}$ has a monodromy action of $\pi_{1}\left(\mathbf{G}_{\mathbf{m}}\right)$; this action is one of the maps on the cone of the above triangle induced by the monodromy action on $R \psi_{f}$, but as this is not functorial, one should consult the real definition in 7]. (given for the algebraic nearby cycles, but see also the second exposé).

Lemma 1.1. There exists a unique decomposition of $R \psi_{f}$ as $R \psi_{f}^{\text {un }} \oplus R \psi_{f}^{\neq 1}$, where for any choice of generator $t$ of $\pi_{1}\left(\mathbf{G}_{\mathbf{m}}\right), 1-t$ acts nilpotently on $R \psi_{f}^{\text {un }}\left(A_{U}^{\bullet}\right)$ for any complex $A_{U}^{\bullet}$ and is an automorphism of $R \psi_{f}^{\neq 1}$.

The part $R \psi_{f}^{\text {un }}$ is called the functor of unipotent nearby cycles. 
Proof. To start, we observe that for any sheaf $\mathcal{F}$ on $U$, we have $\psi_{f}(\mathcal{F})=H^{0} R \psi_{f}(\mathcal{F})$, and so if $\mathcal{F}$ is constructible, by the constructibility of nearby cycles so is $\psi_{f}(\mathcal{F})$; thus, for any open $V \subset X, \psi_{f}(\mathcal{F})(V)$ is finite-dimensional. Let $\psi_{f}^{\text {un }} \subset \psi_{f}$ be the subfunctor such that for any sheaf $\mathcal{F}$ on $U, \psi_{f}^{\text {un }}(\mathcal{F})$ is the subsheaf of $\psi_{f}(\mathcal{F})$ in which $1-t$ is nilpotent, so for each $V, \psi_{f}^{\text {un }}(\mathcal{F})(V)$ is the generalized eigenspace of $t$ with eigenvalue 1 .

Therefore it is actually a direct summand; we recall the general argument which works over any field $k$. If $T$ is an endomorphism of a finite-dimensional vector space $M$, we view $M$ as a $k[x]$-module with $x$ acting as $T$. By the classification of modules over a principal ideal domain, we have $M \cong \bigoplus k[x] / p(x)$ for certain polynomials $p(x)$. The generalized eigenspace with eigenvalue 1 is then the sum of those pieces for which $p(x)$ is a power of $1-x$, and the remaining summands are a $T$-invariant complement in which $1-T$ acts invertibly. As the image of $(1-T)^{n}(n \gg 0)$, this complement is functorial in the category of finite-dimensional $k[x]$-modules and so we have the same decomposition in sheaves of finite-dimensional $k[x]$-modules.

Specializing again to the present situation, we get a decomposition $\psi_{f}(\mathcal{F}) \cong$ $\psi_{f}^{\text {un }}(\mathcal{F}) \oplus \psi_{f}^{\neq 1}$ (this is the definition of $\psi_{f}^{\neq 1}$ ). Both summands are a fortiori leftexact functors and taking derived functors, we obtain a decomposition:

$$
R \psi_{f} \cong R \psi_{f}^{\text {un }} \oplus R \psi_{f}^{\neq 1} .
$$

Since $1-t$ is nilpotent on any $\psi_{f}^{\text {un }}(\mathcal{F})$, we may apply $\psi_{f}^{\text {un }}$ to any constructible complex of injectives, thus computing $R \psi_{f}^{\text {un }}\left(A_{U}^{\bullet}\right)$ for any complex $A_{U}^{\bullet}$, and conclude that $1-t$ is nilpotent on each such; by general principles it is invertible on $R \psi_{f}^{\neq 1}$. This is what we want.

Uniqueness of the decomposition is clear; indeed, if in any category with a zero object we have objects $x$ and $y$ together with endomorphisms $N$ and $I$ respectively such that $N$ is nilpotent and $I$ invertible, then any map $g: x \rightarrow y$ intertwining $N$ and $I$ is zero: we have $g N=I g$, so $g=I^{-1} g N=I^{-2} g N^{2}=\cdots=I^{-n} g N^{n}=0$ if $N^{n}=0$. For morphisms $y \rightarrow x$ we work in the opposite category. In particular, if we have

$$
R \psi_{f} \cong F \oplus G
$$

as a sum of two functors as in the statement of the lemma, then the identity map on $R \psi_{f}$ has no $G$-component on $R \psi_{f}^{\text {un }}$ and no $F$-component on $R \psi_{f}^{\neq 1}$, and so induces isomorphisms $R \psi_{f}^{\text {un }} \cong F$ and $R \psi_{f}^{\neq 1} \cong G$.

We note that this lemma is a special case of Lemma 4.2 when the field of coefficients is algebraically closed. However, this decomposition is defined over any field.

There is a triangle, functorial in $A_{X}^{\bullet}$,

$$
i^{*} j_{*} j^{*} A_{X}^{\bullet} \rightarrow R \psi_{f}\left(j^{*} A_{X}^{\bullet} \stackrel{1-t}{\longrightarrow} R \psi_{f}\left(j^{*} A_{X}^{\bullet}\right)\right.
$$

(see 5 . Prop. 1.1; 13 , eq. (5.88)]) which, taking $A_{U}^{\bullet}=j^{*} A_{X}^{\bullet}$ and inserting $R \psi_{f}^{\text {un }}$ because the monodromy acts trivially on the first term, gives the extremely important (for us) triangle

$$
i^{*} j_{*} A_{U}^{\bullet} \rightarrow R \psi_{f}^{\mathrm{un}}\left(A_{U}^{\bullet} \stackrel{1-t}{\longrightarrow} R \psi_{f}^{\mathrm{un}}\left(A_{U}^{\bullet}\right) \rightarrow\right.
$$


We also have a unipotent part of the vanishing cycles functor $R \phi_{f}$, and, again since the monodromy acts trivially on $i^{*} A_{X}^{\bullet}$, a corresponding triangle

$$
i^{*} A_{X}^{\bullet} \rightarrow R \psi_{f}^{\mathrm{un}}\left(A_{X}^{\bullet}\right) \rightarrow R \phi_{f}^{\mathrm{un}}\left(A_{X}^{\bullet}\right) \rightarrow
$$

If $\mathcal{L}$ is any locally constant sheaf on $\mathbf{G}_{\mathbf{m}}$ with underlying vector space $L$ and unipotent monodromy, then $R \psi_{f}^{\text {un }}\left(A_{U}^{\bullet} \otimes f^{*} \mathcal{L}\right) \cong R \psi_{f}^{\text {un }}\left(A_{U}^{\bullet}\right) \otimes L$, where $\pi_{1}\left(\mathbf{G}_{\mathbf{m}}\right)$ acts on the tensor product by acting on each factor (since $\mathcal{L}$ is trivialized on $\widetilde{\mathbf{G}_{\mathbf{m}}}$ ).

We note the following fact, crucial to all computations in this paper:

$$
j \text { is an affine morphism. }
$$

Indeed, $Z$ is cut out by a single algebraic equation. Although when $Z$ is any Cartier divisor it is still locally defined by equations $f$ and the inclusion $j$ of its complement is again an affine morphism, it is not necessarily possible to glue nearby cycles which are locally defined as above; c.f. [6, Remark 5.5.4]; an explicit example will appear in 8. However, it follows from Corollary 2.7 that when $\mathcal{M}$ is a perverse sheaf and $R \psi_{f}^{\mathrm{un}}(\mathcal{M})$ has trivial monodromy, it is in fact independent of $f$ and gluing is indeed possible.

Triangle (4) already implies that nearby cycles preserve perverse sheaves.

Lemma 1.2. The functor $R \psi_{f}^{\text {un }}[-1]$ sends ${ }^{p} \mathbf{D}(U)^{\leqslant 0}$ to ${ }^{p} \mathbf{D}(Z)^{\leqslant 0}$ and takes $\mathbf{M}(U)$ to $\mathbf{M}(Z)$.

Proof. Since $j$ is affine and an open immersion, $j_{*}$ and $j$ ! are t-exact, so for any $A_{U}^{\bullet} \in{ }^{p} \mathbf{D}(U)^{\leqslant 0}, i^{*} j_{*} A_{U}^{\bullet}=\operatorname{Cone}\left(j_{!} A_{U}^{\bullet} \rightarrow j_{*} A_{U}^{\bullet}\right)$ is in ${ }^{p} \mathbf{D}(Z)^{\leqslant 0}$. If we apply the long exact sequence of perverse cohomology to triangle (4), we therefore get in nonnegative degrees:

$$
\begin{aligned}
{ }^{p} H^{0}\left(R \psi_{f}^{\mathrm{un}} A_{U}^{\bullet}\right) \stackrel{1-t}{\longrightarrow}{ }^{p} H^{0}\left(R \psi_{f}^{\mathrm{un}} A_{U}^{\bullet}\right) \rightarrow\left(0={ }^{p} H^{1}\left(i^{*} j_{*} A_{U}^{\bullet}\right)\right) \rightarrow \\
{ }^{p} H^{1}\left(R \psi_{f}^{\mathrm{un}} A_{U}^{\bullet}\right) \stackrel{1-t}{\longrightarrow}{ }^{p} H^{1}\left(R \psi_{f}^{\mathrm{un}} A_{U}^{\bullet}\right) \rightarrow\left(0={ }^{p} H^{2}\left(i^{*} j_{*} A_{U}^{\bullet}\right)\right) \rightarrow \cdots
\end{aligned}
$$

For $i \geq 0$, the map ${ }^{p} H^{i}\left(R \psi_{f}^{\text {un }} A_{U}^{\bullet}\right) \rightarrow{ }^{p} H^{i}\left(R \psi_{f}^{\text {un }} A_{U}^{\bullet}\right)$ is both given by a nilpotent operator and is surjective, so zero. It follows that $R \psi_{f}^{\text {un }}\left(A_{U}^{\bullet}\right) \in{ }^{p} \mathbf{D}(Z)^{\leqslant-1}$, as promised.

Now let $\mathcal{M} \in \mathbf{M}(U)$ be a perverse sheaf. Then $i^{*} j_{*} \mathcal{M} \in{ }^{p} \mathbf{D}(Z)^{[-1,0]}$ since its perverse cohomology sheaves are the kernel and cokernel of the map $j_{!} \mathcal{M} \rightarrow j_{*} \mathcal{M}$. In degrees $\leq-2$, then, we have

$$
\begin{aligned}
\cdots \rightarrow\left(0={ }^{p} H^{-3}\right. & \left.\left(i^{*} j_{*} \mathcal{M}\right)\right) \rightarrow{ }^{p} H^{-3}\left(R \psi_{f}^{\text {un }} \mathcal{M}\right) \stackrel{1-t}{\longrightarrow}{ }^{p} H^{-3}\left(R \psi_{f}^{\text {un }} \mathcal{M}\right) \rightarrow \\
\left(0={ }^{p} H^{-2}\left(i^{*} j_{*} \mathcal{M}\right)\right) & \rightarrow{ }^{p} H^{-2}\left(R \psi_{f}^{\text {un }} \mathcal{M}\right) \stackrel{1-t}{\longrightarrow}{ }^{p} H^{-2}\left(R \psi_{f}^{\text {un }} \mathcal{M}\right)
\end{aligned}
$$

This means that for $i \leq-2$, all the maps $1-t$ are injective and nilpotent, hence zero. Thus $R \psi_{f}^{\text {un }}(\mathcal{M}) \in{ }^{p} \mathbf{D}(Z)^{-1}$, as desired. $\Psi_{f}^{\text {un }}$.

Since $R \psi_{f}^{\text {un }}[-1]$ acts on perverse sheaves, we will give it the abbreviated notation

\section{Construction of the unipotent nearby CyCles functor}

Let $L^{a}$ be the vector space of dimension $a \geq 0$ together with the action of a matrix $J^{a}=\left[\delta_{i j}-\delta_{i, j-1}\right]$, a unipotent (variant of a) Jordan block of dimension $a$. Let $\mathcal{L}^{a}$ be the locally constant sheaf on $\mathbf{G}_{\mathbf{m}}$ whose underlying space is $L^{a}$ and in whose monodromy action a (hereafter fixed choice of) generator $t$ of $\pi_{1}\left(\mathbf{G}_{\mathbf{m}}\right)$ acts 
by $J^{a}$. Since it is locally free, it is flat, so we will write $\otimes$ rather than $\otimes^{L}$ in tensor products with it (actually, with $f^{*} \mathcal{L}^{a}$ ). It has the following self-duality properties, where $\check{\mathcal{L}}^{a}=\mathcal{H} \operatorname{om}\left(\mathcal{L}^{a}, \underline{\mathbb{C}}\right)$ is the dual local system and $\left(\mathcal{L}^{a}\right)^{-1}$ is the local system in whose monodromy $t$ acts by $\left(J^{a}\right)^{-1}$ :

Lemma 2.1. We have $\mathcal{L}^{a} \cong \check{\mathcal{L}}^{a} \cong\left(\mathcal{L}^{a}\right)^{-1}$, and $\mathbb{D}\left(A_{U}^{\bullet} \otimes f^{*} \mathcal{L}^{a}\right) \cong \mathbb{D}\left(A_{U}^{\bullet}\right) \otimes f^{*} \mathcal{L}^{a}$ for $A_{U}^{\bullet} \in \mathbf{D}(U)$.

Proof. Since $\check{\mathcal{L}}^{a}$ is the local system with vector space the dual $\check{L}^{a}$ and monodromy $\left(\left(J^{a}\right)^{t}\right)^{-1}$, its monodromy is again unipotent with a single Jordan block of length $a$. We fix in $L^{a}$ the given basis $\vec{e}_{1}, \ldots, \vec{e}_{a}$ associated to $J$, and in $\check{L}^{a}$ we choose a generalized eigenbasis $\check{f}_{1}, \ldots, \check{f}_{n}$ in which $\left(\left(J^{a}\right)^{t}\right)^{-1}$ has the matrix $J^{a}$, so that sending $\vec{e}_{i} \mapsto \check{f}_{i}$ identifies $J^{a}$ with $\left(\left(J^{a}\right)^{t}\right)^{-1}$ and thus induces the desired map of local systems. The same proof shows that $\mathcal{L}^{a} \cong\left(\mathcal{L}^{a}\right)^{-1}$.

In general, then, we construct an isomorphism:

$$
\mathbb{D}\left(A_{U}^{\bullet}\right) \otimes f^{*} \mathcal{L}^{a} \stackrel{\sim}{\longrightarrow} \mathbb{D}\left(A_{U}^{\bullet} \otimes f^{*} \mathcal{L}^{a}\right),
$$

where

$$
\begin{aligned}
& \mathbb{D}\left(A_{U}^{\bullet}\right) \otimes f^{*} \mathcal{L}^{a}=R \mathcal{H} \operatorname{om}\left(A_{U}^{\bullet}, \mathcal{D}^{\bullet}\right) \stackrel{L}{\otimes} f^{*} \mathcal{L}^{a}, \\
& \mathbb{D}\left(A_{U}^{\bullet} \otimes f^{*} \mathcal{L}^{a}\right)=R \mathcal{H} \operatorname{om}\left(A_{U}^{\bullet} \stackrel{L}{\otimes} f^{*} \mathcal{L}^{a}, \mathcal{D}_{U}^{\bullet}\right)=R \mathcal{H} \operatorname{om}\left(A_{U}^{\bullet}, \mathbb{D} f^{*} \mathcal{L}^{a}\right),
\end{aligned}
$$

by constructing a map

$$
R \mathcal{H} \operatorname{om}\left(A_{U}^{\bullet}, \mathcal{D}_{U}^{\bullet}\right) \stackrel{L}{\otimes} f^{*} \mathcal{L}^{a} \rightarrow R \mathcal{H} \operatorname{om}\left(A_{U}^{\bullet}, \mathbb{D} f^{*} \mathcal{L}^{a}\right) .
$$

Such a map can be obtained by applying $\left(\otimes^{L}, R \mathcal{H}\right.$ om)-adjunction to a map:

$$
\begin{aligned}
R \mathcal{H o m}\left(A_{U}^{\bullet}, \mathcal{D}_{U}^{\bullet}\right) \rightarrow R \mathcal{H o m}\left(f^{*} \mathcal{L}^{a}, R \mathcal{H o m}\left(A_{U}^{\bullet}, \mathbb{D} f^{*} \mathcal{L}^{a}\right)\right) \\
\quad=R \mathcal{H o m}\left(A_{U}^{\bullet}, R \mathcal{H} \operatorname{om}\left(f^{*} \mathcal{L}^{a}, \mathbb{D} f^{*} \mathcal{L}^{a}\right)\right) .
\end{aligned}
$$

Since $\mathbb{D}$ exchanges ! and $*$, we have $\mathbb{D} f^{*} \mathcal{L}^{a}=f^{!} \mathbb{D} \mathcal{L}^{a}$. By the property $(2)$ of $f^{!}$, we have

$$
R \mathcal{H} \operatorname{om}\left(f^{*} \mathcal{L}^{a}, f^{!} \mathbb{D} \mathcal{L}^{a}\right)=f^{!} R \mathcal{H} \operatorname{om}\left(\mathcal{L}^{a}, \mathbb{D} \mathcal{L}^{a}\right)
$$

Note also that $\mathcal{D}_{U}^{\bullet}=f^{!} \mathcal{D}_{\mathbf{G}_{\mathrm{m}}}^{\bullet}$ by definition and therefore the map (7) can be constructed by applying $R \mathcal{H} \operatorname{om}\left(A_{U}^{\bullet}, f^{!} \bullet\right)$ to a certain map on $\mathbf{G}_{\mathbf{m}}$ :

$$
\mathcal{D}_{\mathbf{G}_{\mathrm{m}}}^{\bullet} \rightarrow R \mathcal{H} \operatorname{om}\left(\mathcal{L}^{a}, \mathbb{D} \mathcal{L}^{a}\right)=\mathbb{D}\left(\mathcal{L}^{a} \stackrel{L}{\otimes} \mathcal{L}^{a}\right) .
$$

This map, in turn, is obtained by first replacing the $\otimes^{L}$ with $\otimes$ (since $\mathcal{L}^{a}$ is locally free) and applying $\mathbb{D}$ to the pairing

$$
\mathcal{L}^{a} \otimes \mathcal{L}^{a} \rightarrow \underline{\mathbb{C}}
$$

given by the isomorphism $\mathcal{L}^{a} \cong \check{\mathcal{L}}^{a}$ described in the first paragraph. Thus, locally (6) is the tautological isomorphism $\left(\mathbb{D} A_{U}^{\bullet}\right)^{\oplus a} \cong \mathbb{D}\left(A_{U}^{\bullet}\right)^{\oplus a}$. Since it is a local isomorphism, it is an isomorphism.

In the rest of this section, $\mathcal{M}$ is any object of $\mathbf{M}(U)$. The following construction is Beilinson's definition of the unipotent nearby cycles:

Proposition 2.2. Let $\alpha^{a}: j_{!}\left(\mathcal{M} \otimes f^{*} \mathcal{L}^{a}\right) \rightarrow j_{*}\left(\mathcal{M} \otimes f^{*} \mathcal{L}^{a}\right)$ be the natural map. Then there is an inclusion $\operatorname{ker}\left(\alpha^{a}\right) \hookrightarrow \Psi_{f}^{\text {un }}(\mathcal{M})$, identifying the actions of $\pi_{1}\left(\mathbf{G}_{\mathbf{m}}\right)$, which is an isomorphism for all sufficiently large $a$. (In fact, it suffices to take $a$ large enough that $(1-t)^{a}$ annihilates $\Psi_{f}^{\text {un }}(\mathcal{M})$.) 
Proof. We know by Lemma 1.2 that $\Psi_{f}^{\text {un }}(\bullet)$ is a perverse sheaf, so taking together the triangle (4) with $A_{U}^{\bullet}=\mathcal{M} \otimes f^{*} \mathcal{L}^{a}$ and exact sequence (1) with $A_{X}^{\bullet}=j_{*} A_{U}^{\bullet}$, we see that $\operatorname{ker} \alpha=\operatorname{ker}(1-t)$, where $1-t$ is the map appearing in the former triangle shifted by -1 . We also have

$$
\Psi_{f}^{\mathrm{un}}\left(\mathcal{M} \otimes f^{*} \mathcal{L}^{a}\right) \cong \Psi_{f}^{\mathrm{un}}(\mathcal{M}) \otimes L^{a} \cong \bigoplus_{i=1}^{a} \Psi_{f}^{\mathrm{un}}(\mathcal{M})_{(i)},
$$

where the $i$ 'th coordinate of the action of $t$ is $t_{(i)}-t_{(i+1)}$, with $t_{(i)}$ the copy of $t \in \pi_{1}\left(\mathbf{G}_{\mathbf{m}}\right)$ acting on $\Psi_{f}^{\mathrm{un}}(\mathcal{M})$ considered as the $i$ 'th summand. That is, using elements, $\left(x_{1}, \ldots, x_{n}\right) \in \Psi_{f}^{\mathrm{un}}\left(\mathcal{M} \otimes f^{*} \mathcal{L}^{a}\right)$ is sent by $t$ to $\left(t x_{1}-t x_{2}, t x_{2}-t x_{3}, \ldots, t x_{n}\right)$. Thus, for an element of $\operatorname{ker}(1-t)$, we have $x_{i+1}=\left(1-t^{-1}\right) x_{i}$, or:

$$
x_{i}=\left(1-t^{-1}\right)^{i-1} x_{1} \quad-t\left(1-t^{-1}\right)^{a} x_{1}=(1-t) x_{n}=0 .
$$

If we define a map $u: \Psi_{f}^{\mathrm{un}}(\mathcal{M}) \rightarrow \Psi_{f}^{\mathrm{un}}\left(\mathcal{M} \otimes f^{*} \mathcal{L}^{a}\right)$ by sending the element $x=x_{1}$ to the coordinates $x_{i}$ defined by the first formula above, then $u$ is injective and its image contains $\operatorname{ker}(1-t)$ (namely, that subspace satisfying the second equation). Since $1-t$ (hence $1-t^{-1}$ ) is nilpotent on $\Psi_{f}^{\text {un }}(\mathcal{M})$, for $a$ sufficiently large, $\operatorname{im}(u)=$ $\operatorname{ker}(1-t)$. We claim that $u$ intertwines the actions of $t^{-1}$ and $J^{a}$ :

$$
\begin{aligned}
J^{a} u(x)=\left(x-\left(1-t^{-1}\right) x,\left(1-t^{-1}\right) x+\right. & \left.\left(1-t^{-1}\right)^{2} x, \ldots\right) \\
& =\left(t^{-1} x,\left(1-t^{-1}\right) t^{-1} x, \ldots\right)=u\left(t^{-1} x\right) .
\end{aligned}
$$

Finally, we employ the isomorphism $\mathcal{L}^{a} \cong\left(\mathcal{L}^{a}\right)^{-1}$ of Lemma 2.1 to give an automorphism of $\operatorname{ker}\left(\alpha^{a}\right) \subset j !\left(\mathcal{M} \otimes f^{*} \mathcal{L}^{a}\right)$ intertwining $J^{a}$ and $\left(J^{a}\right)^{-1}$.

Corollary 2.3. There exists an integer $N$ such that $(1-t)^{N}$ annihilates both $\operatorname{ker} \alpha^{a}$ and $\operatorname{coker} \alpha^{a}$ for all $a$.

Proof. By Proposition 2.2 the kernel is contained in $\Psi_{f}^{\text {un }}(\mathcal{M})$ and thus annihilated by that power of $1-t$ which annihilates the nearby cycles. Temporarily let $\alpha^{a}=$ $\alpha_{\mathcal{M}}^{a}$; then $\mathbb{D}\left(\alpha_{\mathcal{M}}^{a}\right)=\alpha_{\mathbb{D} \mathcal{M}}^{a}$, so $\operatorname{coker}\left(\alpha_{\mathcal{M}}^{a}\right)=\mathbb{D} \operatorname{ker}\left(\alpha_{\mathbb{D} \mathcal{M}}^{a}\right)$ is again annihilated by some $(1-t)^{N}$.

In preparation for the next section, we give a generalization of this construction. For each $a, b \geq 0$ there is a natural short exact sequence

$$
0 \rightarrow \mathcal{L}^{a} \stackrel{g^{a, b}}{\longrightarrow} \mathcal{L}^{a+b} \stackrel{g^{a+b,-a}}{\longrightarrow} \mathcal{L}^{b} \rightarrow 0
$$

that is, for any $r \in \mathbb{Z}, g^{a, r}$ sends $\mathcal{L}^{a}$ to the first $a$ coordinates of $\mathcal{L}^{a+r}$ if $r \geq 0$, and to the quotient $\mathcal{L}^{a-(-r)}$ given by collapsing the first $-r$ coordinates if $-r \geq 0$ (that is, $r \leq 0)$ and $a+r \geq 0$. This sequence respects the action of $\pi_{1}\left(\mathbf{G}_{\mathbf{m}}\right)$ on the terms and, via Lemma 2.1, the $(a, b)$ sequence is dual to the $(b, a)$ sequence.

Let $\mathcal{M} \in \mathbf{M}(U)$; then we have induced maps on the tensor products:

$$
g_{\mathcal{M}}^{a, r}=\operatorname{id} \otimes g^{a, r}: \mathcal{M} \otimes f^{*} \mathcal{L}^{a} \rightarrow \mathcal{M} \otimes f^{*} \mathcal{L}^{a+r}
$$

(we will often omit the subscript $\mathcal{M}$ when no confusion is possible). By Lemma 2.1. these satisfy

$$
\mathbb{D} g_{\mathcal{M}}^{a, r}=g_{\mathbb{D} \mathcal{M}}^{a+r,-r}
$$

Note that since the $\mathcal{L}^{a}$ are locally free, the $g_{\mathcal{M}}^{a, r}$ are all injective when $r \geq 0$ and surjective when $r \leq 0$. Let $r \in \mathbb{Z}$ and set

$$
\alpha^{a, r}=j_{*}\left(g^{a, r}\right) \circ \alpha^{a}=\alpha^{a+r} \circ j_{!}\left(g^{a, r}\right): j_{!}\left(\mathcal{M} \otimes f^{*} \mathcal{L}^{a}\right) \rightarrow j_{*}\left(\mathcal{M} \otimes f^{*} \mathcal{L}^{a+r}\right) .
$$


We will use the following self-evident properties of the $g^{a, r}$ :

Lemma 2.4. The $g^{a, r}$ satisfy:

(1) When $a+r \geq 0$, we have $g^{a, r} \circ g^{a+r,-r}=(1-t)^{|r|}$.

(2) When $r$ and $s$ have the same sign and $a+r+s \geq 0$, we have $g^{a, r+s}=$ $g^{a+r, s} \circ g^{a, r}$.

(3) Let $r \geq 0, a \geq r$; then we have:

$$
\operatorname{ker}(1-t)^{r}=\operatorname{ker}\left(g_{\mathcal{M}}^{a,-r}\right) \cong \mathcal{M} \otimes f^{*} \mathcal{L}^{r}, \quad \operatorname{im}(1-t)^{r}=\operatorname{im}\left(g_{\mathcal{M}}^{a-r, r}\right) \cong \mathcal{M} \otimes f^{*} \mathcal{L}^{a-r} .
$$

Finally, by Corollary 2.3 and (3), for $r \geq 0,(1-t)^{N+r}$ annihilates $\operatorname{ker}\left(\alpha^{a,-r}\right)$ and $\operatorname{coker}\left(\alpha^{a, r}\right)$.

From now on, we will assume $r \geq 0$.

Proposition 2.5. For $a \gg 0$, the natural maps $j_{!}\left(g^{a, 1}\right)$ and $j_{*}\left(g^{a+r,-1}\right)$ respectively induce isomorphisms

$$
\operatorname{ker}\left(\alpha^{a,-r}\right) \stackrel{\sim}{\longrightarrow} \operatorname{ker}\left(\alpha^{a+1,-r}\right) \quad \operatorname{coker}\left(\alpha^{a, r}\right) \stackrel{\sim}{\longrightarrow} \operatorname{coker}\left(\alpha^{a-1, r}\right)
$$

and $j_{!}\left(g^{a, r}\right)$ and $j_{*}\left(g^{a+r,-r}\right)$ induce isomorphisms

$$
\operatorname{ker}\left(\alpha^{a, r}\right) \stackrel{\sim}{\rightarrow} \operatorname{ker}\left(\alpha^{a+r}\right) \quad \operatorname{coker}\left(\alpha^{a+r}\right) \stackrel{\sim}{\rightarrow} \operatorname{coker}\left(\alpha^{a+r,-r}\right)
$$

Proof. Using the maps $j_{!}\left(g^{a, 1}\right)$ and $j_{*}\left(g^{a-r, 1}\right)$ we get a square which, using Lemma $2.4[2$, we verify is commutative:

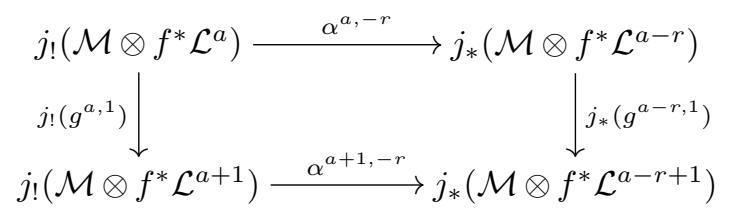

showing that $j_{!}\left(g^{a, 1}\right)$ induces a map on kernels. Since it is injective, we get a long sequence of inclusions of kernels:

$$
\cdots \subset \operatorname{ker} \alpha^{a-1,-r} \subset \operatorname{ker} \alpha^{a,-r} \subset \operatorname{ker} \alpha^{a+1,-r} \subset \cdots .
$$

By Lemma 2.4, each kernel is annihilated by $(1-t)^{N+r}$, whose kernel is (for $a \geq$ $N+r)$ the perverse sheaf $j_{!}\left(\mathcal{M} \otimes f^{*} \mathcal{L}^{N+r}\right)$; thus, this sequence is contained in this sheaf. Since perverse sheaves are noetherian, this chain must have a maximum, so the kernels stabilize. For the cokernels, we apply (8) to the argument of Corollary 2.3. (One can also argue directly using the artinian property of perverse sheaves.)

For the second statement concerning kernels, since $(1-t)^{N}$ annihilates $\operatorname{ker}\left(\alpha^{a+r}\right)$, for $a \geq N$ it is contained in $\operatorname{im}\left(g^{a, r}\right)$, and therefore by definition in $\operatorname{ker}\left(\alpha^{a, r}\right)$. The statement on cokernels is again obtained by dualization and (8). (A direct argument employing a diagram chase is also possible, using the fact that (1$t)^{N} \operatorname{coker}\left(\alpha^{a+r}\right)=0$.)

Departing slightly from Beilinson's notation, we denote these stable kernels and cokernels $\operatorname{ker} \alpha^{\infty,-r}$ and coker $\alpha^{\infty, r}$ for $r \geq 0$; when $r=0$ we drop it.

Proposition 2.6. There is a natural isomorphism $\operatorname{ker} \alpha^{\infty,-r} \stackrel{\sim}{\rightarrow} \operatorname{coker} \alpha^{\infty, r}$. 
Proof. Consider the map of short exact sequences for any $a$ and any $b \geq r$ (to eliminate clutter we have not written the superscripts on the maps $g$ ):

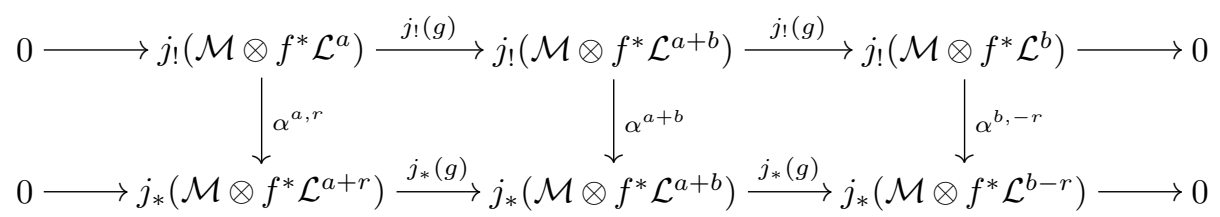

By the snake lemma, we have an exact sequence of kernels and cokernels:

$$
\begin{aligned}
0 \rightarrow \operatorname{ker}\left(\alpha^{a, r}\right) \rightarrow \operatorname{ker}\left(\alpha^{a+b}\right) \rightarrow \operatorname{ker}\left(\alpha^{b,-r}\right) & \stackrel{\gamma^{a, b ; r}}{\longrightarrow} \operatorname{coker}\left(\alpha^{a, r}\right) \\
& \rightarrow \operatorname{coker}\left(\alpha^{a+b}\right) \rightarrow \operatorname{coker}\left(\alpha^{b,-r}\right) \rightarrow 0 .
\end{aligned}
$$

If $a, b \gg 0$, then the first and last maps are, by the second part of Proposition 2.5. isomorphisms. Therefore $\gamma^{a, b ; r}$ is an isomorphism. Since the long exact sequence of cohomology (9) is natural, we see that $\gamma^{a, b ; r}$ is independent of $a$ and $b$ in the sense of the proposition:

$$
\gamma^{a, b+1 ; r} \circ j_{!}\left(g^{b, 1}\right)=\gamma^{a, b ; r} \quad j_{*}\left(g^{a+1,-1}\right) \circ \gamma^{a+1, b ; r}=\gamma^{a, b ; r}
$$

where the requisite commutative diagrams are produced using Lemma 2.4 1 22). For the same reason, $\gamma^{a, b ; r}$ is a natural transformation between the two functors

$$
\mathcal{M} \mapsto \operatorname{ker}\left(\alpha_{\mathcal{M}}^{b,-r}\right), \quad \mathcal{M} \mapsto \operatorname{coker}\left(\alpha_{\mathcal{M}}^{a, r}\right),
$$

Because they are equal, we will give a single name $\Pi_{f}^{r}(\mathcal{M})=\operatorname{ker}\left(\alpha^{\infty,-r}\right) \cong$ $\operatorname{coker}\left(\alpha^{\infty, r}\right)$ to the stable kernel and cokernel. These are thus exact functors, and by definition of $\alpha^{a, r}$ and (8), they commute with duality: $\mathbb{D} \Pi_{f}^{r}(\mathcal{M}) \cong \Pi_{f}^{r}(\mathbb{D} \mathcal{M})$. From Proposition 2.2 we conclude:

Corollary 2.7. For $a \gg 0$ we have $\operatorname{ker}\left(\alpha^{a}\right) \cong \Psi_{f}^{\mathrm{un}}(\mathcal{M}) \cong \operatorname{coker}\left(\alpha^{a}\right)$, and thus an isomorphism

$$
\mathbb{D} \Psi_{f}^{\mathrm{un}}(\mathcal{M}) \cong \Psi_{f}^{\mathrm{un}}(\mathbb{D} \mathcal{M})
$$

which is natural in the perverse sheaf $\mathcal{M}$. A more effective, equivalent construction is obtained as follows: suppose $(1-t)^{N}$ annihilates $\Psi_{f}^{\text {un }}(\mathcal{M})$. Then we have by $(3)$

$$
\Psi_{f}^{\mathrm{un}}(\mathcal{M})=i^{*} j_{! *}\left(\mathcal{M} \otimes f^{*} \mathcal{L}^{N}\right)[-1]=i^{!} j_{! *}\left(\mathcal{M} \otimes f^{*} \mathcal{L}^{N}\right)[1] .
$$

Conversely, if these equations hold, then of course $(1-t)^{N}$ annihilates $\Psi_{f}^{\text {un }}(\mathcal{M})$.

\section{VANishing CYCles AND GLUING}

We will refer to $\Pi_{f}^{1}$ as $\Xi_{f}^{\text {un }}$, which Beilinson calls the "maximal extension functor" and denotes without the superscript. Although there is no independent, nonunipotent analogue, we have chosen to use this notation to match that for the nearby and (upcoming) vanishing cycles functors, which do have such analogues.

Proposition 3.1. There are two natural exact sequences exchanged by duality and $\mathcal{M} \leftrightarrow \mathbb{D} \mathcal{M}$ :

$$
\begin{aligned}
0 & \rightarrow j_{!}(\mathcal{M}) \stackrel{\alpha_{-}}{\longrightarrow} \Xi_{f}^{\text {un }}(\mathcal{M}) \stackrel{\beta_{-}}{\longrightarrow} \Psi_{f}^{\text {un }}(\mathcal{M}) \rightarrow 0 \\
0 & \rightarrow \Psi_{f}^{\text {un }}(\mathcal{M}) \stackrel{\beta_{+}}{\longrightarrow} \Xi_{f}^{\text {un }}(\mathcal{M}) \stackrel{\alpha_{+}}{\longrightarrow} j_{*}(\mathcal{M}) \rightarrow 0,
\end{aligned}
$$

where $\alpha_{+} \circ \alpha_{-}=\alpha$ and $\beta_{-} \circ \beta_{+}=1-t$. 
Proof. These sequences are, respectively, the last and first halves of (9). For the first one, take $b=r$, so $g^{b,-r}=0$ and therefore $\alpha^{b,-r}=0$; we get an exact sequence $0 \rightarrow \operatorname{ker}\left(\alpha^{a, r}\right) \rightarrow \operatorname{ker}\left(\alpha^{a+r}\right) \rightarrow j_{!}\left(\mathcal{M} \otimes f^{*} \mathcal{L}^{r}\right) \stackrel{\gamma^{a, r ; r}}{\longrightarrow} \operatorname{coker}\left(\alpha^{a, r}\right) \rightarrow \operatorname{coker}\left(\alpha^{a+r}\right) \rightarrow 0$.

For $a \gg 0$, by the second part of Proposition 2.5 the first map is an isomorphism, and for $r=1$ we obtain the first short exact sequence from the remaining three terms above. For the second short exact sequence, we apply the same reasoning to (9) with $a=0$ and then $r=1$, with $b \gg 0$ :

$$
0 \rightarrow \operatorname{ker}\left(\alpha^{b}\right) \rightarrow \operatorname{ker}\left(\alpha^{b,-r}\right) \stackrel{\gamma^{0, b ; r}}{\longrightarrow} j_{*}\left(\mathcal{M} \otimes f^{*} \mathcal{L}^{r}\right) \rightarrow \operatorname{coker}\left(\alpha^{b}\right) \rightarrow \operatorname{coker}\left(\alpha^{b,-r}\right) \rightarrow 0 .
$$

It is obvious from these constructions and (8) that the two short exact sequences are exchanged by duality. To show that $\alpha_{+} \circ \alpha_{-}=\mathrm{id}$ and $\beta_{-} \circ \beta_{+}=1-t$, we identify these maps in the above sequences and rewrite the claims as:

$$
\left.\left(\gamma^{0, b ; 1} \circ\left(\gamma^{a, b ; 1}\right)^{-1} \circ \gamma^{a, 1 ; 1}\right)\right|_{U}=\mathrm{id},\left.\quad \gamma^{a, b ; 1}\right|_{\operatorname{ker}\left(\alpha^{b}\right)} \bmod \operatorname{im}\left(\alpha^{a+1}\right)=(1-t) \gamma^{a+1, b ; 0} \text {. }
$$

For both, we use the fact that since $\left.\alpha^{a}\right|_{U}=\mathrm{id}$, we have $\left.\gamma^{a, b ; r}\right|_{U}=\left(g^{a+b,-a}\right.$ 。 $\left.g^{a+r, b-r}\right)^{-1}$, as constructed in the familiar proof of the snake lemma, with the inverse interpreted as a multi-valued pullback. Then the claims are equivalent to

$$
\begin{aligned}
g^{a+b,-a} \circ g^{a+1, b-1} & =g^{1, b-1} \circ g^{a+1,-a} \\
g^{a+b+1,-a-1} \circ g^{a+1, b} & =(1-t) g^{a+b,-a} g^{a+1, b-1}
\end{aligned}
$$

which follow from Lemma 2.4 12 .

The remainder of the paper is simply what Beilinson calls "linear algebra" (one might argue that this has already been the case for most of the preceding). Take $\mathcal{M}=j^{*} \mathcal{F}$ for a perverse sheaf $\mathcal{F} \in \mathbf{M}(X)$ in the above exact sequences. From the maps in these two sequences we can form a complex:

$$
j ! j^{*} \mathcal{F} \stackrel{\left(\alpha_{-}, \gamma_{-}\right)}{\longrightarrow} \Xi_{f}^{\text {un }}\left(j^{*} \mathcal{F}\right) \oplus \mathcal{F} \stackrel{\left(\alpha_{+},-\gamma_{+}\right)}{\longrightarrow} j_{*} j^{*} \mathcal{F},
$$

where $\gamma_{-}: j_{!} j^{*}(\mathcal{F}) \rightarrow \mathcal{F}$ and $\gamma_{+}: \mathcal{F} \rightarrow j_{*} j^{*}(\mathcal{F})$ are defined by the left- and rightadjunctions $\left(j_{!}, j^{*}\right)$ and $\left(j^{*}, j_{*}\right)$ and the property that $j^{*}\left(\gamma_{-}\right)=j^{*}\left(\gamma_{+}\right)=\mathrm{id}$.

Proposition 3.2. The complex $(10)$ is in fact a complex; let $\Phi_{f}^{\text {un }}(\mathcal{F})$ be its cohomology sheaf. Then $\Phi_{f}^{\text {un }}$ is an exact functor $\mathbf{M}(X) \rightarrow \mathbf{M}(Z)$, and there are maps $u, v$ such that $v \circ u=1-t$ as in the following diagram:

$$
\Psi_{f}^{\mathrm{un}}\left(j^{*} \mathcal{F}\right) \stackrel{u}{\rightarrow} \Phi_{f}^{\mathrm{un}}(\mathcal{F}) \stackrel{v}{\rightarrow} \Psi_{f}^{\mathrm{un}}\left(j^{*} \mathcal{F}\right) .
$$

Proof. That (10) is a complex amounts to showing that $\gamma_{+} \circ \gamma_{-}=\alpha=\alpha_{+} \circ \alpha_{-}$, which is true by definition of the $\gamma_{ \pm}$and adjunction. To show that $\Phi_{f}^{\text {un }}$ is exact, suppose we have $0 \rightarrow \mathcal{F}_{1} \rightarrow \mathcal{F}_{2} \rightarrow \mathcal{F}_{3} \rightarrow 0$, so that we get a short exact sequence of complexes

$$
0 \rightarrow C^{\bullet}\left(\mathcal{F}_{1}\right) \rightarrow C^{\bullet}\left(\mathcal{F}_{2}\right) \rightarrow C^{\bullet}\left(\mathcal{F}_{3}\right) \rightarrow 0
$$

where by $C^{\bullet}(\mathcal{F})$ we have denoted the complex (10) padded with zeroes on both sides. Note that since $\alpha_{-}$is injective and $\alpha_{+}$surjective, $C^{\bullet}(\mathcal{F})$ fails to be exact only at the middle term. Therefore we have a long exact sequence of cohomology sheaves:

$\cdots\left(0=H^{-1} C^{\bullet}\left(\mathcal{F}_{3}\right)\right) \rightarrow \Phi_{f}^{\mathrm{un}}\left(\mathcal{F}_{1}\right) \rightarrow \Phi_{f}^{\mathrm{un}}\left(\mathcal{F}_{2}\right) \rightarrow \Phi_{f}^{\mathrm{un}}\left(\mathcal{F}_{3}\right) \rightarrow\left(0=H^{1}\left(C^{\bullet}\left(\mathcal{F}_{1}\right)\right)\right) \cdots$ which shows that $\Phi_{f}^{\text {un }}$ is functorial and an exact functor. 
If we apply $j^{*}$ to $(10)$ it becomes simply $\left(\right.$ with $\left.j^{*} \mathcal{F}=\mathcal{M}\right)$

$$
\mathcal{M} \stackrel{\text { (id,id) }}{\longrightarrow} \mathcal{M} \oplus \mathcal{M} \stackrel{\text { (id,-id) }}{\longrightarrow} \mathcal{M}
$$

which is actually exact, so $j^{*} \Phi_{f}^{\text {un }}(\mathcal{F})=0$; i.e. $\Phi_{f}^{\text {un }}(\mathcal{F})$ is supported on $Z$. Finally, to define $u$ and $v$, let pr: $\Xi_{f}^{\text {un }}\left(j^{*} \mathcal{F}\right) \oplus \mathcal{F} \rightarrow \Xi_{f}^{\text {un }}\left(j^{*} \mathcal{F}\right)$, and set $u=\left(\beta_{+}, 0\right)$ in coordinates, and $v=\beta_{-} \circ$ pr. Since $\beta_{-} \circ \alpha_{-}=0, v$ factors through $\Phi_{f}^{\text {un }}(\mathcal{F})$, and we have $v \circ u=\beta_{-} \circ \beta_{+}=1-t$ by Proposition 3.1

Define a vanishing cycles gluing data for $f$ to be a quadruple $\left(\mathcal{F}_{U}, \mathcal{F}_{Z}, u, v\right)$ as in Proposition 3.2 for any $\mathcal{F} \in \mathbf{M}(X)$, the quadruple $F_{f}(\mathcal{F})=\left(j^{*} \mathcal{F}, \Phi_{f}^{\text {un }}(\mathcal{F}), u, v\right)$ is such data. Let $\mathbf{M}_{f}(U, Z)$ be the category of gluing data; then $F_{f}: \mathbf{M}(X) \rightarrow$ $\mathbf{M}_{f}(U, Z)$ is a functor. Conversely, given a vanishing cycles data

$$
\Psi_{f}^{\mathrm{un}}\left(\mathcal{F}_{U}\right) \stackrel{u}{\rightarrow} \mathcal{F}_{Z} \stackrel{v}{\rightarrow} \Psi_{f}^{\mathrm{un}}\left(\mathcal{F}_{U}\right),
$$

we can form the complex

$$
\Psi_{f}^{\mathrm{un}}\left(\mathcal{F}_{U}\right) \stackrel{\left(\beta_{+}, u\right)}{\longrightarrow} \Xi_{f}^{\mathrm{un}}\left(\mathcal{F}_{U}\right) \oplus \mathcal{F}_{Z} \stackrel{\left(\beta_{-},-v\right)}{\longrightarrow} \Psi_{f}^{\mathrm{un}}\left(\mathcal{F}_{U}\right)
$$

since $v \circ u=1-t=\beta_{-} \circ \beta_{+}$, and let $G_{f}\left(\mathcal{F}_{U}, \mathcal{F}_{Z}, u, v\right)$ be its cohomology sheaf.

Beilinson gives an elegant framework for proving the equivalence of (10) and (11) in 1. Appendix]. Rather than proving Theorem 3.6 directly, we present his technique (with slightly modified terminology).

Definition 3.3. Let a diad be a complex of the form

$$
D^{\bullet}=\left(\mathcal{F}_{L} \stackrel{L=\left(a_{L}, b_{L}\right)}{\longrightarrow} \mathcal{A} \oplus \mathcal{B} \stackrel{R=\left(a_{R}, b_{R}\right)}{\longrightarrow} \mathcal{F}_{R}\right)
$$

in which $a_{L}$ is injective and $a_{R}$ is surjective (so it is exact on the ends). Let the category of diads be denoted $\mathbf{M}_{2}$. Let a triad be a short exact sequence of the form

$$
S=\left(0 \rightarrow \mathcal{F}_{-} \stackrel{\left(c_{-}, d_{-}^{1}, d_{-}^{2}\right)}{\longrightarrow} \mathcal{A} \oplus \mathcal{B}^{1} \oplus \mathcal{B}^{2} \stackrel{\left(c_{+}, d_{+}^{1}, d_{+}^{2}\right)}{\longrightarrow} \mathcal{F}_{+} \rightarrow 0\right)
$$

in which both $\left(c_{-}, d_{-}^{i}\right): \mathcal{F}_{-} \rightarrow \mathcal{A} \oplus \mathcal{B}^{i}$ are injections and both $\left(c_{+}, d_{+}^{i}\right): \mathcal{A} \oplus \mathcal{B}^{i} \rightarrow \mathcal{F}_{+}$ are surjections. Let the category of triads be denoted $\mathbf{M}_{3}$; it has a reflection functor $r: \mathbf{M}_{3} \rightarrow \mathbf{M}_{3}$ which invokes the natural symmetry $1 \leftrightarrow 2$, and is an involution.

We can define a map $T: \mathbf{M}_{2} \rightarrow \mathbf{M}_{3}$ by setting

$$
T(D)=\left(0 \rightarrow \operatorname{ker}(R) \stackrel{\left(\iota_{A}, \iota_{B}, h\right)}{\longrightarrow} \mathcal{A} \oplus \mathcal{B} \oplus H\left(D^{\bullet}\right) \stackrel{\left(\pi_{A}, \pi_{B},-k\right)}{\longrightarrow} \operatorname{coker}(L) \rightarrow 0\right),
$$

where the natural inclusion/projection (resp. projection/inclusion) are called:

$$
\operatorname{ker}(R) \stackrel{\iota=\left(\iota_{A}, \iota_{B}\right)}{\longrightarrow} \mathcal{A} \oplus \mathcal{B} \stackrel{\pi=\left(\pi_{A}, \pi_{B}\right)}{\longrightarrow} \operatorname{coker}(L), \quad \operatorname{ker}(R) \stackrel{h}{\rightarrow} H\left(D^{\bullet}\right) \stackrel{k}{\rightarrow} \operatorname{coker}(L)
$$

(note $\pi \circ \iota=k \circ h$ ). We define the inverse $T^{-1}$ by the formula

$$
T^{-1}(S)=\left(\operatorname{ker}\left(d_{-}^{2}\right) \stackrel{\left(c_{-}, d_{-}^{1}\right)}{\longrightarrow} \mathcal{A} \oplus \mathcal{B}^{1} \rightarrow \operatorname{coker}\left(c_{-}, d_{-}^{1}\right)\right) .
$$

Lemma 3.4. The functors $T, T^{-1}$ are mutually inverse equivalences of $\mathbf{M}_{2}$ with $\mathbf{M}_{3}$. 
Proof. Before beginning the verification of the many necessary facts, we observe that the property of a sequence $S$ as above being in $\mathbf{M}_{3}$ is equivalent to the following diagram being cartesian

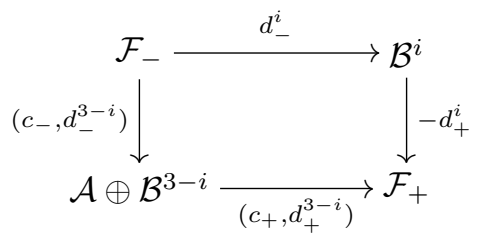

and the following smaller sequence being exact

$$
0 \rightarrow \mathcal{F}_{-} \stackrel{\left(c_{-}, d_{-}^{i}\right)}{\longrightarrow} \mathcal{A} \oplus \mathcal{B}^{i} \stackrel{\left(c_{+}, d_{+}^{i}\right)}{\longrightarrow} \mathcal{F}_{+} \rightarrow 0
$$

for $i=1,2$. Indeed, for (12), the diagram is cartesian if and only if $S$ is exact in the middle, and for (13), the arrows are respectively injective and surjective by hypothesis if $S \in \mathbf{M}_{3}$, while exactness in the middle follows from that of $S$. For readability, we continue the proof as several sub-lemmas.

$T\left(D^{\bullet}\right) \in \mathbf{M}_{3}$ : It is easily verified that $T\left(D^{\bullet}\right)$ is an exact sequence. To see that $\left(\pi_{A},-k\right)$ is surjective and $\left(\iota_{A}, h\right)$ injective, we consider the cartesian diagrams
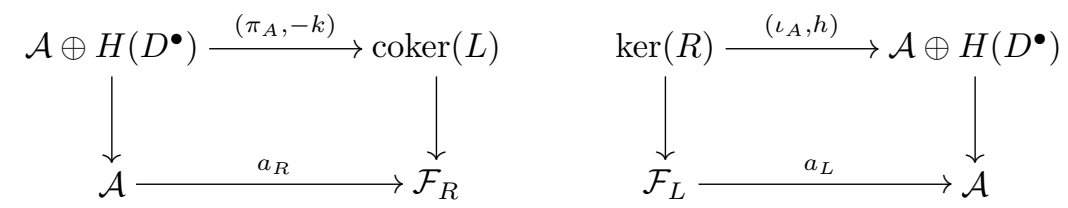

and use that $a_{R}$ is surjective and $a_{L}$ is injective. Likewise, $\left(\pi_{B},-k\right)$ is surjective and $\left(\iota_{B}, h\right)$ is injective.

$T^{-1}(S) \in \mathbf{M}_{2}$ : Clearly, $T^{-1}(S)$ is a complex, since the sequence $\mathcal{F}_{1} \rightarrow \mathcal{A} \oplus \mathcal{B}^{1} \rightarrow$ $\operatorname{coker}\left(c_{-}, d_{-}^{1}\right)$ is exact (hence a complex). Since $\left(c_{-}, d_{-}^{2}\right)$ is injective by hypothesis, $\left.c_{-}\right|_{\operatorname{ker}\left(d_{-}^{2}\right)}$ is injective. We must show that $\mathcal{A} \rightarrow \operatorname{coker}\left(c_{-}, d_{-}^{1}\right)$ is surjective, where by (13) with $i=1$ we have $\operatorname{coker}\left(c_{-}, d_{-}^{1}\right)=\mathcal{F}_{+}$; consider the diagram

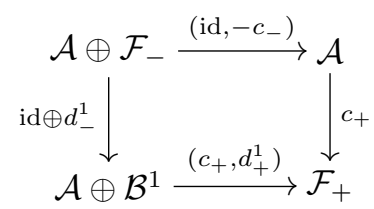

which is cartesian by exactness of (13). Since the bottom arrow is a surjection, for $c_{+}$to be a surjection it suffices to show that the left arrow is. By (12) with $i=1$, $d_{-}^{1}$ is a surjection since the bottom arrow there is a surjection by hypothesis.

$T^{-1} \circ T \cong \mathrm{id}$ : Its $\mathcal{F}_{L}$ is $\operatorname{ker}(h)=\operatorname{im}(L)=\mathcal{F}_{L} ;$ its $\mathcal{A}$ and $\mathcal{B}$ are indeed $\mathcal{A}$ and $\mathcal{B}$, and its $\mathcal{F}_{R}$ is $\operatorname{coker}(\iota)=\mathcal{F}_{R}$; one checks quickly that the maps are right as well.

$T \circ T^{-1} \cong$ id: Its $\mathcal{F}_{-}$is $\operatorname{ker}\left(\mathcal{A} \oplus \mathcal{B}^{1} \rightarrow \operatorname{coker}\left(c_{-}, d_{-}^{1}\right)\right)=\mathcal{F}_{-}$since $\left(c_{-}, d_{-}^{1}\right)$ is an injection; its $\mathcal{A}$ and $\mathcal{B}^{1}$ are obviously the original $\mathcal{A}$ and $\mathcal{B}^{1}$. The small sequence (13) with $i=1$ shows that $\mathcal{F}_{+}$is correct as well. Finally, for $\mathcal{B}^{2}$, we must show that $\mathcal{F}_{-} / \operatorname{ker}\left(d_{-}^{2}\right)=\mathcal{B}^{2}$, or in other words, that $d_{-}^{2}$ is surjective, which follows from (12) with $i=2$. 
Clearly, both of the complexes (10) and (11) are diads. Comparing them, we find that the construction of the latter is given by:

Corollary 3.5. The reflection functor on a diad is the complex

$$
r\left(D^{\bullet}\right)=\left(\operatorname{ker}\left(a_{R}\right) \stackrel{\left(a_{L}^{\prime}, b_{L}^{\prime}\right)}{\longrightarrow} \mathcal{A} \oplus H\left(D^{\bullet}\right) \stackrel{\left(a_{R}^{\prime}, b_{R}^{\prime}\right)}{\longrightarrow} \operatorname{coker}\left(a_{L}\right)\right),
$$

where $a_{L}^{\prime}$ is the natural inclusion and $a_{R}^{\prime}$ the natural projection, $b_{L}^{\prime}=h \circ\left(a_{L}^{\prime}, 0\right)$, and $b_{R}^{\prime}$ factors $-k$ through $\operatorname{coker}\left(a_{L}\right) \subset \operatorname{coker}(L)$.

Proof. That is, $T^{-1} r T\left(D^{\bullet}\right)=r\left(D^{\bullet}\right)$ as defined above. We need to show that $\operatorname{ker}\left(\iota_{B}\right)=\operatorname{ker}\left(a_{R}\right)$ and $\operatorname{coker}\left(\iota_{A}, h\right)=\operatorname{coker}\left(a_{L}\right)$, and prove the identities of the morphisms. The first is easily verified directly, considering both as subobjects of $\mathcal{A} \oplus \mathcal{B}$, while for the second, we assert that the map

$$
(\text { id, } 0): \mathcal{A} \rightarrow \mathcal{A} \oplus H\left(D^{\bullet}\right)
$$

induces the desired isomorphism from the latter to the former. To show that it identifies $\operatorname{im}\left(a_{L}\right)$ with $\operatorname{im}\left(\iota_{A}, h\right)$, it suffices to check that the following diagram is cartesian:

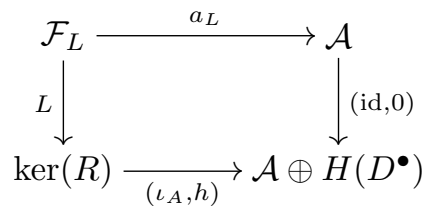

which follows from the definition of $H\left(D^{\bullet}\right)=\operatorname{ker}(R) / \operatorname{im}(R)$. The identities of $a_{L}^{\prime}$, $b_{L}^{\prime}$, and $a_{R}^{\prime}$ are clear from these constructions, while for $b_{R}^{\prime}$ it is fastest to chase the above diagram.

Theorem 3.6. The gluing category $\mathbf{M}_{f}(U, Z)$ is abelian; $F_{f}: \mathbf{M}(X) \rightarrow \mathbf{M}_{f}(U, Z)$ and $G_{f}: \mathbf{M}_{f}(U, Z) \rightarrow \mathbf{M}(X)$ are mutually inverse exact functors, and so $\mathbf{M}_{f}(U, Z)$ is equivalent to $\mathbf{M}(X)$.

Proof. That $\mathbf{M}_{f}(U, Z)$ is abelian amounts to proving that taking coordinatewise kernels and cokernels works. That is, if we have $\left(\mathcal{M}, \mathcal{F}_{Z}, u, v\right)$ and $\left(\mathcal{M}^{\prime}, \mathcal{F}_{Z}^{\prime}, u^{\prime}, v^{\prime}\right)$ with maps $a_{U}: \mathcal{M} \rightarrow \mathcal{M}^{\prime}, a_{Z}: \mathcal{F}_{Z} \rightarrow \mathcal{F}_{Z}^{\prime}$ and such that the following diagram commutes:

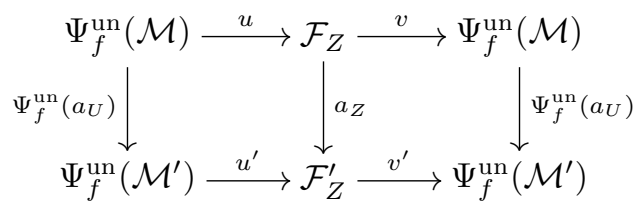

then $\left(\operatorname{ker} a_{U}, \operatorname{ker} a_{Z}, \widetilde{u}, \widetilde{v}\right)$ is a kernel for $\left(a_{U}, a_{V}\right)$, where $\widetilde{u}$ and $\widetilde{v}$ are induced maps; likewise for the cokernel; and we must show that $\left(a_{U}, a_{V}\right)$ is an isomorphism if and only if the kernel and cokernel vanish. The maps $\widetilde{u}$ and $\widetilde{v}$ are constructed from the natural sequence of kernels (or cokernels) in the above diagram, and the exactness of $\Psi_{f}^{\mathrm{un}}$, and once they exist it is obvious from the definition of morphisms in $\mathbf{M}_{f}(U, Z)$ that the desired gluing data is a kernel (resp. cokernel). Since $\mathbf{M}(U)$ and $\mathbf{M}(Z)$ are abelian and kernels and cokernels are taken coordinatewise, the last claim follows.

To show that $F_{f}$ and $G_{f}$ are mutually inverse, we interpret $\mathbf{M}(X)$ and $\mathbf{M}_{f}(U, Z)$ as diad categories in the form given, respectively, by diagrams (10) and (11). The 
reflection functor is given by Corollary 3.5 by Proposition 3.1 and the definition of $\Phi^{\mathrm{un}}$, its value on $(10)$ is that of the functor $F_{f}$. For the same reason, its value on (11) is that of $G_{f}$ interpreted as a complex of type (10) (the $\mathcal{F}$ term is what we have previously called the value of $G_{f}$ ). Since the reflection functor is an involution, $G_{f}$ and $F_{f}$ are mutually inverse.

\section{Comments}

We conclude with some musings on the theory exposited here. In the previous version arXiv:1002.1686v2 of these notes, we gave a substantially different proof of Proposition 2.6 adhering closely to that given in [1, Key Lemma]. As that proof may better illuminate the two-sided limit formalism which we also omit, the curious reader is encouraged to consult it.

The vanishing cycles functor and $\Phi_{f}^{\text {un }}$. The functor $\Phi_{f}^{\text {un }}$, like $\Psi_{f}^{\text {un }}$, has a familiar identity.

Theorem 4.1. There is an isomorphism of functors $\Phi_{f}^{\mathrm{un}} \cong R \phi_{f}^{\mathrm{un}}[-1]$ and a natural distinguished triangle

$$
\Psi_{f}^{\mathrm{un}}\left(j^{*} \mathcal{F}\right) \stackrel{u}{\rightarrow} \Phi_{f}^{\mathrm{un}}(\mathcal{F}) \rightarrow i^{*} \mathcal{F} \rightarrow
$$

isomorphic to that in (5).

Proof. According to the definition of $\Phi_{f}^{\text {un }}$ in Proposition 3.2, we have a short exact sequence and, thus, a corresponding distinguished triangle of the same form:

$$
0 \rightarrow j ! j^{*} \mathcal{F} \rightarrow \operatorname{ker}\left(\alpha_{+},-\gamma_{+}\right) \rightarrow \Phi_{f}^{\text {un }}(\mathcal{F}) \rightarrow 0 .
$$

Since $K=\operatorname{ker}\left(\alpha_{+},-\gamma_{+}\right) \subset \Xi_{f}^{\text {un }}\left(j^{*} \mathcal{F}\right) \oplus \mathcal{F}$, there is a projection map pr: $K \rightarrow \mathcal{F}$ commuting with the inclusion of $j ! j^{*} \mathcal{F}$.

Now we apply the octahedral axiom of triangulated categories as given in 4 . (1.1.7.1)]:

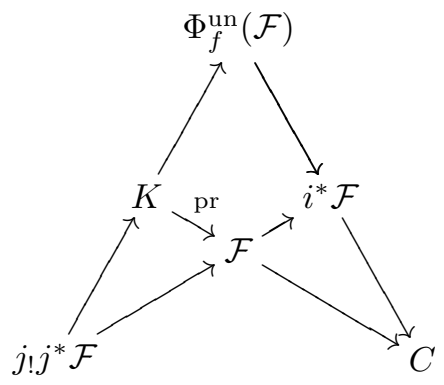

where all the straight lines are distinguished triangles, both the (geometric) triangles are commutative, and the square commutes. It is easy to see that pr must be surjective because $\alpha_{+}$is surjective; thus, since both $K$ and $\mathcal{F}$ are perverse, $C[-1]$ is also perverse, and so we have an exact sequence

$$
0 \rightarrow C[-1] \rightarrow K \stackrel{\mathrm{pr}}{\longrightarrow} \mathcal{F} \rightarrow 0 .
$$

But by definition, $\operatorname{ker}(\operatorname{pr})=\operatorname{ker}\left(\alpha_{+}\right) \oplus 0$, and therefore $C[-1] \cong \Psi_{f}^{\mathrm{un}}\left(j^{*} \mathcal{F}\right)$. Note that the inclusion then becomes the map $u$, as defined in the proof of Proposition 3.2. Rotating the other triangle in the above octahedral diagram, we have

$$
\Psi_{f}^{\mathrm{un}}\left(j^{*} \mathcal{F}\right) \stackrel{u}{\rightarrow} \Phi_{f}^{\mathrm{un}}(\mathcal{F}) \rightarrow i^{*} \mathcal{F} \rightarrow
$$


Comparing with $(5)$, we find that $R \phi_{f}^{\text {un }}(\mathcal{F})[-1] \cong \Phi_{f}^{\text {un }}(\mathcal{F})$ is perverse. Conversely, starting from (5) in place of the above triangle, we conclude by the octahedral axiom that $R \phi_{f}^{\text {un }}(\mathcal{F})[-1]$ is the cohomology of $(10)$ which admits a unique extension to a functor of $\mathcal{F}$ compatible with the octahedral diagram. Therefore, we conclude an isomorphism of functors $\Phi_{f}^{\text {un }} \cong R \phi_{f}^{\text {un }}[-1]$.

The full nearby cycles functor $R \psi_{f}$. As Beilinson observes, the full nearby cycles functor $R \psi_{f}(\mathcal{M})$, for $\mathcal{M} \in \mathbf{M}(U)$, can be recovered from $R \psi_{f}^{\text {un }}$ as applied to variations of $\mathcal{M}$. Here we must assume that the field of coefficients is algebraically closed.

Lemma 4.2. There exists a unique isomorphism of functors $\mathbf{D}(U) \rightarrow \mathbf{D}(Z)$

$$
R \psi_{f}=\bigoplus_{\lambda \in \mathbb{C}^{*}} R \psi_{f}^{\lambda}
$$

where for any constructible complex $A_{U}^{\bullet}$ on $U, \lambda-t$ is nilpotent on $R \psi_{f}^{\lambda}\left(A_{U}^{\bullet}\right)$.

Proof. Simply pursue the line of reasoning in Lemma 1.1 but, since the field of coefficients is algebraically closed, produce the full Jordan decomposition rather than just the unipotent and non-unipotent parts. The lemma can also be deduced from 10, Lemme 3.2.5], which applies to the Jordan decomposition of an endomorphism of any complex in the derived category.

Let $\mathcal{L}_{\lambda}$ be the local system of rank 1 on $\mathbf{G}_{\mathbf{m}}$ with monodromy $\lambda$; then clearly, we have $R \psi_{f}^{\lambda}(\mathcal{M})=R \psi_{f}^{\text {un }}\left(\mathcal{M} \otimes f^{*} \mathcal{L}_{\lambda}^{-1}\right) \otimes L_{\lambda}$, where $t$ acts as $\lambda$ on the one-dimensional vector space $L_{\lambda}$. Substituting into (14), we obtain:

$$
R \psi_{f}(\mathcal{M})=\bigoplus_{\lambda} \Psi_{f}^{\mathrm{un}}\left(\mathcal{M} \otimes f^{*} \mathcal{L}_{\lambda}^{-1}\right) \otimes L_{\lambda}
$$

Thus, Corollary 2.7 gives a procedure for computing the full nearby cycles functor of perverse sheaves, and $R \psi_{f}[-1]$ sends perverse sheaves on $U$ to perverse sheaves on $X$.

Using some general reasoning, we can extend the properties of $\Psi_{f}^{\mathrm{un}}=R \psi_{f}^{\mathrm{un}}[-1]$ from the subcategory of perverse sheaves to the entire derived category. To this end, let $T: \mathbf{C} \rightarrow \mathbf{D}$ be a triangulated functor between triangulated categories with t-structures, and let the respective cores be the abelian categories $\mathbf{A}, \mathbf{B}$. We will assume that the objects of $\mathbf{C}$ are bounded above, meaning that $\mathbf{C}=\bigcup_{b \in \mathbb{Z}} \mathbf{C} \leqslant b$.

Lemma 4.3. Suppose $T$ is right t-exact and that $T \mathbf{A} \subset \mathbf{B}$; then $T$ is t-exact.

Proof. We will show that $T$ commutes with all truncations. Suppose we have an object $x \in \mathbf{C} \leqslant b$, so that there is a distinguished triangle

$$
\tau^{<b} x \rightarrow x \rightarrow \tau^{\geqslant b} x \rightarrow
$$

where by definition, $\tau^{\geqslant b} x=H^{b}(x)[-b] \in \mathbf{A}[-b]$. By hypothesis on $T$, we have $T(x) \in \mathbf{D}^{\leqslant b}, T\left(\tau^{<b} x\right) \in \mathbf{D}^{<b}$, and $T\left(H^{b} x[-b]\right) \in \mathbf{B}[-b] \subset \mathbf{D}^{\geqslant b}$. Since $T$ is triangulated, there is a triangle

$$
T\left(\tau^{<b} x\right) \rightarrow T(x) \rightarrow T\left(H^{b} x[-b]\right) \rightarrow
$$

and therefore, by uniqueness of the truncation triangle, it must be that $T\left(\tau^{<b} x\right)=$ $\tau^{<b} T(x)$. This is under the hypothesis that $x \in \mathbf{C}^{\leqslant b}$; since then $\tau^{<b} x \in \mathbf{C} \leqslant b-1$ and since $\tau^{<b-1} \tau^{<b}=\tau^{<b-1}$, we can apply truncations-by-one repeatedly and conclude that for all $n$, we have $\tau^{\leqslant n} T(x)=T\left(\tau^{\leqslant n} x\right)$. 
Now suppose we have any $x$, and for any $n$ form the distinguished triangle

$$
\tau^{<n} x \rightarrow x \rightarrow \tau^{\geqslant n} \rightarrow
$$

to which we apply $T$. Since $T\left(\tau^{<n} x\right)=\tau^{<n} T(x)$, the cone of the resulting triangle

$$
\tau^{<n} T(x) \rightarrow T(x) \rightarrow T\left(\tau^{\geqslant n} x\right) \rightarrow
$$

must be isomorphic to $\tau^{\geqslant n} T(x)$, by uniqueness of cones and the truncation triangle for $T(x)$. Thus, $\tau^{\geqslant n} T(x)=T\left(\tau^{\geqslant n} x\right)$. Since then $T$ commutes with all trunctions, it is a fortiori t-exact.

Take $T=R \psi_{f}^{\text {un }}[-1]$; by Lemma 1.2, it satisfies the hypothesis of Lemma 4.3. and therefore we conclude:

Theorem 4.4. The functor $R \psi_{f}[-1]$ on the bounded derived category $\mathbf{D}^{b}(X)$ is t-exact for the perverse t-structure. Likewise, $R \phi_{f}[-1]$ is t-exact.

Proof. For the second statement, we must show that $R \phi_{f}[-1]$ is right t-exact and preserves perverse sheaves; the latter claim already follows from Theorem 4.1. For the former, we apply the long exact sequence to the triangle

$$
i^{*} \mathcal{F} \rightarrow R \psi_{f}\left(j^{*} \mathcal{F}\right) \rightarrow R \phi_{f}(\mathcal{F}) \rightarrow
$$

We have $i^{*} \mathcal{F} \in{ }^{p} \mathbf{D}(X)^{[-1,0]}$ because of triangle (1) and we already know that $R \psi_{f}[-1]$ is right t-exact, so the long exact sequence of perverse cohomology shows that ${ }^{p} H^{i}\left(R \phi_{f} \mathcal{F}\right)=0$ when $i \geq 0$, as desired.

We will not prove here that $R \psi_{f}[-1]$ commutes with Verdier duality. This is significantly more difficult since it necessitates enlarging the domain of a certain natural transformation (the map $\gamma^{a, b ; r}$ constructed in Proposition 2.6 from the core of the perverse t-structure to the entire derived category. This involves the interaction with both objects and morphisms:

- The natural maps must be defined for all objects, not just those in $\mathbf{M}(U)$;

- The maps thus obtained must commute with all morphisms, not just those between objects of $\mathbf{M}(U)$.

To see why this is difficult, consider showing merely that the $\gamma^{a, b ; r}$ (and their translates) are natural with respect to maps of the form $g: \mathcal{M} \rightarrow \mathcal{N}[i]$, with $i \in \mathbb{N}$ and $\mathcal{M}, \mathcal{N} \in \mathbf{M}(U)$. Note that the argument given for the naturality of $\gamma^{a, b ; r}$ is not valid in this context, since kernel and cokernel constructions in the abelian category of perverse sheaves are not functorial in the entire derived category.

If $i=1$, this is easy; we necessarily have $\operatorname{Cone}(g) \in \mathbf{M}(U)[1]$, so rotating the distinguished triangle gives a short exact sequence

$$
0 \rightarrow \mathcal{N} \rightarrow \operatorname{Cone}(g)[-1] \rightarrow \mathcal{M} \rightarrow 0 .
$$

Conversely, this sequence constructs the distinguished triangle $\mathcal{M} \rightarrow \mathcal{N}[1] \rightarrow$ Cone $(g)$ by the reverse procedure. Then, applying $\mathbb{D} \Psi_{f}^{\text {un }}$ and $\Psi_{f}^{\text {un }} \mathbb{D}$ to the sequence, we find by naturality of $\gamma^{a, b ; r}$ that there is a commutative diagram of short exact sequences, which implies that $\gamma^{a, b ; r}$ is natural with respect to $g$.

The analogue of this argument for $i>1$ would involve finding a sequence of the form

$$
0 \stackrel{h_{i+1}=0}{\longrightarrow}\left(\mathcal{N}=\mathcal{A}^{-(i+1)}\right) \stackrel{h_{i}}{\longrightarrow} \mathcal{A}^{-i} \rightarrow \cdots \rightarrow \mathcal{A}^{-1} \stackrel{h_{0}}{\longrightarrow}\left(\mathcal{A}^{0}=\mathcal{M}\right) \rightarrow 0
$$


representing $g$. The manner in which such a sequence does represent such a map is clear; we get a collection of short exact sequences representing maps:

$$
0 \rightarrow \operatorname{coker}\left(h_{j+1}\right) \rightarrow \mathcal{A}^{-j} \rightarrow \operatorname{coker}\left(h_{j}\right) \rightarrow 0, \quad g_{j}: \operatorname{coker}\left(h_{j}\right) \rightarrow \operatorname{coker}\left(h_{j+1}\right)[1]
$$

(where $\operatorname{coker}\left(h_{i+1}\right)=\mathcal{N}$ and $\left.\operatorname{coker}\left(h_{1}\right)=\mathcal{M}\right)$, and thus, by composition, a map $g: \mathcal{M} \rightarrow \mathcal{N}[i]$, as desired. This is Yoneda's realization of $\operatorname{Ext}^{i}(\mathcal{M}, \mathcal{N})$; it holds in the derived category of $\mathbf{M}(U)$. It is, however, a nontrivial theorem, proved in 2, that this is the same as $\mathbf{D}(U)$, and in fact it is describing the morphisms that occupies the entirety of the work in that paper. Of course, once we choose to cite this result, it is a trivial consequence of Corollary 2.7 that $R \psi_{f}[-1]$ commutes with $\mathbb{D}$, since it is then the derived functor of a self-dual exact functor on $\mathbf{M}(U)$. Thus, we do not expect that there will be as elementary an argument as for the perversity of nearby cycles.

In the recent preprint 12 , autoduality of the nearby cycles functor is proven in complete generality in the complex analytic setting, and references are given there for prior results and those in the algebraic setting.

The maximal extension functor $\Xi_{f}^{\text {un }}$. We have used the term "maximal extension functor" without explanation (as did Beilinson), but Proposition 3.1 provides sufficient rationale: applying $i^{*}$ to the first one and $i^{!}$to the second one, the long exact sequence of perverse cohomology shows that $i^{*} \Xi_{f}^{\text {un }}(\mathcal{M}) \cong \Psi_{f}^{\text {un }}(\mathcal{M}) \cong i ! \Xi_{f}^{\text {un }}(\mathcal{M})$ are both perverse sheaves, which is as far out (cohomologically) as they can be given that $i^{*}$ is right t-exact and $i^{!}$is left t-exact. This should be compared with the defining property of the "minimal extension" $j_{! *}(\mathcal{M})$, that $i^{*} j_{! *}(\mathcal{M})[-1]$ and $i^{!} j_{!_{*}}(\mathcal{M})[1]$ are perverse, so that it has a minimal presence on $X$ given that it extends $\mathcal{M}$. The condition that $i^{*} \Xi_{f}^{\text {un }}(\mathcal{M})$ and $i^{!} \Xi_{f}^{\text {un }}(\mathcal{M})$ are perverse does not uniquely characterize $\Xi_{f}^{\text {un }}(\mathcal{M})$, as one could add any perverse sheaf supported on $Z$ without changing it, but imposing Proposition 3.1 forbids such a modification. As we will see below, these sequences uniquely determine $\Xi_{f}^{\mathrm{un}}(\mathcal{M})$.

To do so, consider the pair of upper and lower "caps" of an octahedron:
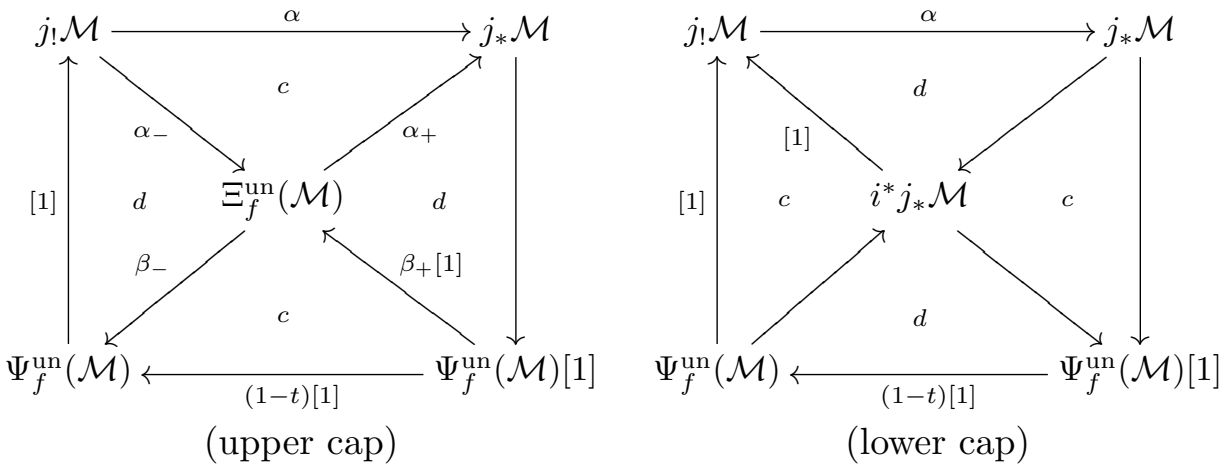

The triangles marked "c" are commutative and those marked "d" are distinguished; the arrows marked [1] have their targets (but not their sources) shifted by 1 . The octahedral axiom states that given any diagram of commutative and distinguished triangles as in (lower cap) we can construct a diagram as in (upper cap) and vice versa $([4, \S 1.1 .6])$. Using these diagrams, we can derive $(4)$ and Proposition 3.1 from each other. This idea is also present in [6, §5.7.2]. 
Proposition 4.5. Suppose we have functors $\Xi_{f}^{\text {un }}$ and $\Psi_{f}^{\text {un }}$ from $\mathbf{M}(U)$ to $\mathbf{M}(X)$, where $\Psi_{f}^{\text {un }}$ has a unipotent action of $\pi_{1}\left(\mathbf{G}_{\mathbf{m}}\right)$, and satisfying Proposition 3.1. Then (4) holds with $R \psi_{f}^{\text {un }}=\Psi_{f}^{\text {un }}[1]$.

Proof. Given Proposition 3.1, each exact sequence there corresponds to a unique distinguished triangle in $\mathbf{D}(X)$ with the same entries; these triangles appear in (upper cap), where the top and bottom maps are $\alpha$ and $(1-t)[1]$ since the triangles containing them are commutative. The octahedral axiom gives us (lower cap), and since the upper triangle is distinguished its cone (the middle term) must necessarily be $i^{*} j_{*} \mathcal{M}$ by (1). Therefore the bottom triangle is (4), as desired. Note that all the interior maps in (lower cap) are uniquely determined, since they correspond to the kernels and cokernels of the maps $\alpha$ and $1-t$ of perverse sheaves in the long exact sequence of cohomology.

Proposition 4.6. Given only the triangle (4) both the functor $\Xi_{f}^{\text {un }}$ and its extension classes in $\operatorname{Ext}^{1}\left(\Psi_{f}^{\text {un }}(\mathcal{M}), j_{!} \mathcal{M}\right)$ and $\operatorname{Ext}^{1}\left(j_{*} \mathcal{M}, \Psi_{f}^{\text {un }}(\mathcal{M})\right)$ can be constructed with Proposition 3.1 satisfied (except for the duality statement). In particular, by Proposition 4.5, $\Xi_{f}^{\text {un }}$ is uniquely determined by Proposition 3.1 .

Proof. Given (4), since we have (1) canonically we can form all the vertices of (lower cap) and both distinguished triangles; the left and right maps are determined by the requirement that the triangles containing them be commutative. The octahedral axiom gives us (upper cap) and $\Xi_{f}^{\text {un }}(\mathcal{M})$, identified at first only as an element of $\mathbf{D}(X)$. From Lemma 1.2 we know that $\Psi_{f}^{\text {un }}(\mathcal{M})$ is perverse; then the long exact sequence of perverse cohomology associated to either distinguished triangle in (upper cap) shows that, in fact, $\Xi_{f}^{\text {un }}(\mathcal{M})$ is perverse, and thus those triangles correspond to exact sequences as in Proposition 3.1. The equations $\alpha_{+} \alpha_{-}=\alpha$ and $\beta_{-} \beta_{+}=1-t$ can then be read off from the commutativity of the upper and lower triangles. Since the vertical arrows come from (lower cap), these distinguished triangles are uniquely determined up to isomorphism fixing $j_{*, !} \mathcal{M}$ and $\Psi_{f}^{\text {un }}(\mathcal{M})$, as desired.

The identity of $\Xi_{f}^{\text {un }}$ is somewhat mysterious, but can be made precise using the gluing category. These computations are also given in [6, Example 5.7.8].

Proposition 4.7. For any perverse sheaf $\mathcal{M} \in \mathbf{M}(U)$, we have the following correspondences via the gluing construction:

$$
\begin{array}{ll}
j_{!}(\mathcal{M})=\left(\mathcal{M}, \Psi_{f}^{\text {un }}(\mathcal{M}), \text { id }, 1-t\right) & j_{! *}(\mathcal{M})=(\mathcal{M}, \operatorname{im}(1-t), 1-t, \text { incl }) \\
j_{*}(\mathcal{M})=\left(\mathcal{M}, \Psi_{f}^{\text {un }}(\mathcal{M}), 1-t, \text { id }\right) & \Xi_{f}^{\text {un }}(\mathcal{M})=\left(\mathcal{M}, \Psi_{f}^{\text {un }}\left(\mathcal{M} \otimes f^{*} \mathcal{L}^{2}\right), u, v\right) ;
\end{array}
$$

where $\alpha: j_{!}(\mathcal{M}) \rightarrow j_{*}(\mathcal{M})$ is the map (id, $\left.1-t\right)$ in the gluing category; in $j_{! *}(\mathcal{M})$, we mean $\operatorname{im}(1-t) \subset \Psi_{f}^{\text {un }}(\mathcal{M})$; in $\Xi_{f}^{\text {un }}(\mathcal{M})$, taking $\Psi_{f}^{\text {un }}\left(\mathcal{M} \otimes f^{*} \mathcal{L}^{a}\right)=\Psi_{f}^{\text {un }}(\mathcal{M}) \oplus$ $\Psi_{f}^{\mathrm{un}}(\mathcal{M})$, we have $u=(\mathrm{id}, 1-t)$ and $v=\operatorname{pr}_{2}$.

Proof. Using the triangle of Theorem 4.1, we have

$$
\Psi_{f}^{\mathrm{un}}\left(j^{*} j_{!} \mathcal{M}\right) \rightarrow \Phi_{f}^{\mathrm{un}}\left(j_{!} \mathcal{M}\right) \rightarrow i^{*} j_{!}(\mathcal{M}) \rightarrow
$$

and since $i^{*} j_{!}=0$, we get an isomorphism $\Phi_{f}^{\text {un }}\left(j_{!} \mathcal{M}\right) \cong \Psi_{f}^{\text {un }}(\mathcal{M})$; dualizing, we have $\Phi_{f}^{\mathrm{un}}\left(j_{*} \mathcal{M}\right) \cong \Psi_{f}^{\mathrm{un}}(\mathcal{M})$ also. Since $u$ is the first map in this triangle, under this identification we have $u=\mathrm{id}$, and therefore $v=1-t$ since $v \circ u=1-t$. This gives the quadruple for $j_{!}(\mathcal{M})$; for $j_{*}(\mathcal{M})$, we dualize, since $u$ and $v$ are dual by their 
definition in Propositions 3.2 and 3.1 . That the natural map is given by (id, $1-t$ ) follows from the fact that this does define a map $j_{!}(\mathcal{M}) \rightarrow j_{*}(\mathcal{M})$ in the gluing category, and that its restriction to $U$ is the identity.

For $j_{! *}(\mathcal{M})$, we use the fact that it is the image of the natural map $\alpha: j_{!}(\mathcal{M}) \rightarrow$ $j_{*}(\mathcal{M})$; having already identified all the parties, this is clear from the quadruples just obtained.

For the identification of $\Xi_{f}^{\text {un }}(\mathcal{M})$, obviously, $v \circ u=1-t$; more importantly, $u$ is injective and $v$ surjective. Then the pair of exact sequences in Proposition 3.1 can be described on quadruples as being trivial over $U$, and over $Z$ the maps $\alpha_{-}$and $\alpha_{+}$are described by the following maps of quadruples:

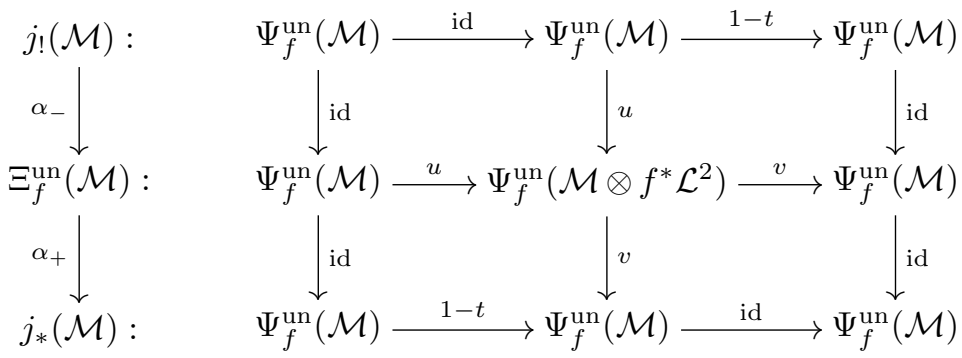

We take $\beta_{-}$and $\beta_{+}$to be the maps whose $Z$-parts (the $U$-parts are zero) are:

$$
\beta_{-}(y, z)=(1-t) y-z \quad \beta_{+}(x)=(x, 0) .
$$

Then it is clear from the definitions of $u$ and $v$ that we obtain the sequences of Proposition 3.1. by the uniqueness part of Proposition 4.6, this uniquely determines $\Xi_{f}^{\text {un }}(\mathcal{M})$, completing the proof.

Since the entirety of Section 3 follows only from Proposition 3.1, Propositions 4.5 and 4.6 show that the constructions of Section 2 are irrelevant for constructing the gluing functor. Their purpose, as is evident from the order we have chosen for the theorems, is to exhibit the autoduality of $\Psi_{f}^{\mathrm{un}}$ and $\Xi_{f}^{\mathrm{un}}$ (and, thus, $\Phi_{f}^{\mathrm{un}}$ ). However, Beilinson's development has an aesthetic virtue (over just using the above short proof of Proposition 3.1): once Lemma 1.2 is proven, the entire theory takes place within the abelian category of perverse sheaves. In addition, Corollary 2.7 is an ingeniously elementary, insightful, and more useful definition of a functor whose actual definition is quite obscure. 


\section{REFERENCES}

[1] Alexander Beilinson, How to glue perverse sheaves, $K$-theory, arithmetic and geometry (Moscow, 1984), Lecture Notes in Math., vol. 1289, Springer, Berlin, 1987, pp. 42-51. MR923134 (89b:14028)

[2] _ On the derived category of perverse sheaves, $K$-theory, arithmetic and geometry (Moscow, 1984), Lecture Notes in Math., vol. 1289, Springer, Berlin, 1987, pp. 27-41. MR923133 (89b:14027)

[3] Alexander Beilinson and Joseph Bernstein, A proof of Jantzen conjectures, I. M. Gelfand Seminar, Adv. Soviet Math., vol. 16, Amer. Math. Soc., Providence, RI, 1993, pp. 1-50. MR1237825 (95a:22022)

[4] Alexander Beilinson, Joseph Bernstein, and Pierre Deligne, Faisceaux pervers, Analysis and topology on singular spaces, I (Luminy, 1981), Astérisque, vol. 100, Soc. Math. France, Paris, 1982, pp. 5-171 (French). MR751966 (86g:32015)

[5] Jean-Luc Brylinski, Transformations canoniques, dualité projective, théorie de Lefschetz, transformations de Fourier et sommes trigonométriques, Astérisque 140-141 (1986), 3134, 251 (French, with English summary). Géométrie et analyse microlocales. MR864073 (88j:32013)

[6] Mark Andrea A. de Cataldo and Luca Migliorini, The decomposition theorem, perverse sheaves and the topology of algebraic maps, Bull. Amer. Math. Soc. (N.S.) 46 (2009), no. 4, 535-633, DOI 10.1090/S0273-0979-09-01260-9 MR2525735

[7] Pierre Deligne, Le formalisme des cycles évanescents, SGA VII (Exposés XIII, XIV), available at http://www.msri.org/publications/books/sga/sga/pdf/sga7-2.pdf

[8] Fouad El Zein, Trang Lê Dung, and Luca Migliorini, A topological construction of the weight filtration. To appear.

[9] Sergei I. Gelfand and Yuri I. Manin, Methods of homological algebra, 2nd ed., Springer Monographs in Mathematics, Springer-Verlag, Berlin, 2003. MR1950475 (2003m:18001)

[10] Gérard Laumon and Bao Châu Ngô, Le lemme fondamental pour les groupes unitaires, Ann. of Math. (2) 168 (2008), no. 2, 477-573, DOI 0.4007/annals.2008.168.477 (French, with English summary). MR2434884 (2009i:22022)

[11] Sam Lichtenstein, Vanishing cycles for algebraic D-modules, Bachelor's thesis, Harvard University, 2009, http://math.harvard.edu/ gaitsgde/grad_2009/Lichtenstein(2009).pdf

[12] David B. Massey, Natural Commuting of Vanishing Cycles and the Verdier Dual, available at arXiv:0908.2799v1.

[13] Jörg Schürmann, Topology of singular spaces and constructible sheaves, Instytut Matematyczny Polskiej Akademii Nauk. Monografie Matematyczne (New Series) [Mathematics Institute of the Polish Academy of Sciences. Mathematical Monographs (New Series)], vol. 63, Birkhäuser Verlag, Basel, 2003. MR2031639 (2005f:32053)

E-mail address: ryanr@math.harvard.edu 